\title{
Ad Honorem Charles Fefferman
}

Communicated by Stephen Kennedy and Steven J. Miller

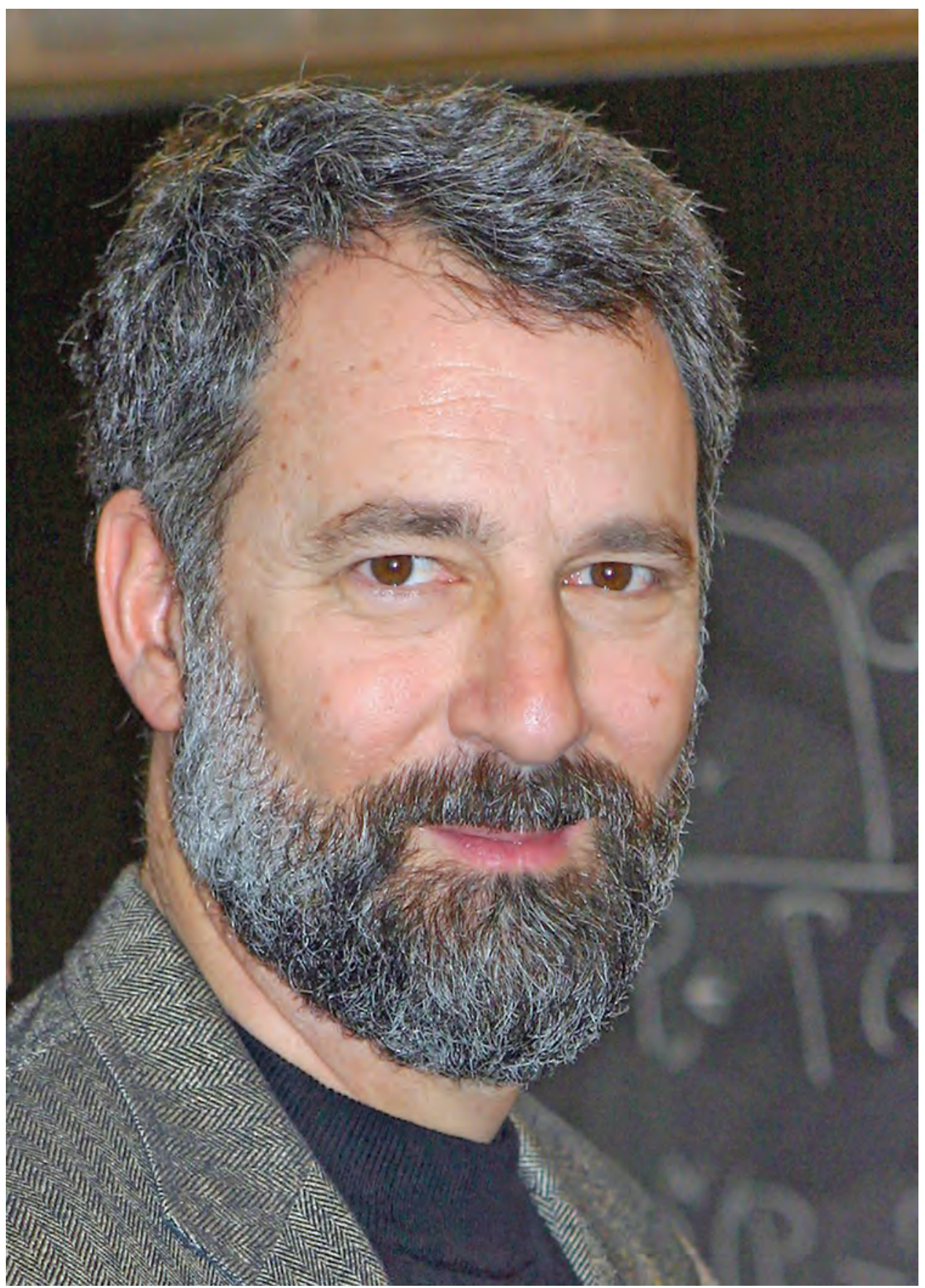

Charles Fefferman circa 2005. 


\section{Antonio Córdoba}

\section{Introduction}

The prestigious Wolf Prize of 2017 has been awarded to Charles Fefferman, ex aequo with Richard Schoen. Charles Fefferman (Charlie) is a mathematician of the first rank whose outstanding findings, both classical and revolutionary, have inspired further research by many others. He is one of the most accomplished and versatile mathematicians of all time, having so far contributed with fundamental results to harmonic analysis, linear PDEs, several complex variables, conformal geometry, quantum mechanics, fluid mechanics, and Whitney's theory, together with more sporadic incursions into other subjects such as neural networks, financial mathematics, and crystallography.

I have requested the help of a distinguished group of his friends and collaborators to provide reflections on Fefferman's contributions to their respective fields. Before reading their remarks, it will be interesting to hear from Charlie himself: "Problems seem to select me! It's just so exciting. A problem sort of chooses you, and you can't stop thinking about it. At first, you try something, and it doesn't work. You get clobbered! You try something else and get clobbered again! Eventually you get some insights and things begin to come together. Everything starts to move. Everyday things can look different. It's very exciting. Eventually you manage to solve it all, and that's a great feeling!"

Born on April 18, 1949, Fefferman was a child prodigy who at the age of seventeen graduated from the University of Maryland, where he received a joint bachelor's degree in mathematics and physics. In 1969 he gained his PhD at Princeton University under the supervision of Eli Stein. In 1971, at the University of Chicago, he became the youngest full professor at any US college or university, a fact that merited his appearance in Time and Newsweek magazines in that same year.

Charlie returned to Princeton University in the fall of 1974, where since then he has pursued his mathematical career. In 1975 he and his wife, Julie, got married and went on to have two daughters, Nina and Lainie. Nina is a computational biologist who applies mathematical models to complex biological systems, while Lainie is a composer and holds a $\mathrm{PhD}$ in musical composition from Princeton University. Charlie has a brother, Robert, who is also a mathematician and professor at the University of Chicago. Julie has this to say about her husband: "When Charlie was young, he fell in love: (1) with painting and art and (2) with math. He says that for a while the two were

Antonio Córdoba is the director of the Mathematics Institute (ICMAT) and professor of mathematics at the Autonomous University of Madrid. His email address is antoni o. cordoba@uam. es.

For permission to reprint this article, please contact:

reprint-permission@ams .org.

DOI: http://dx.doi.org/10.1090/noti1606

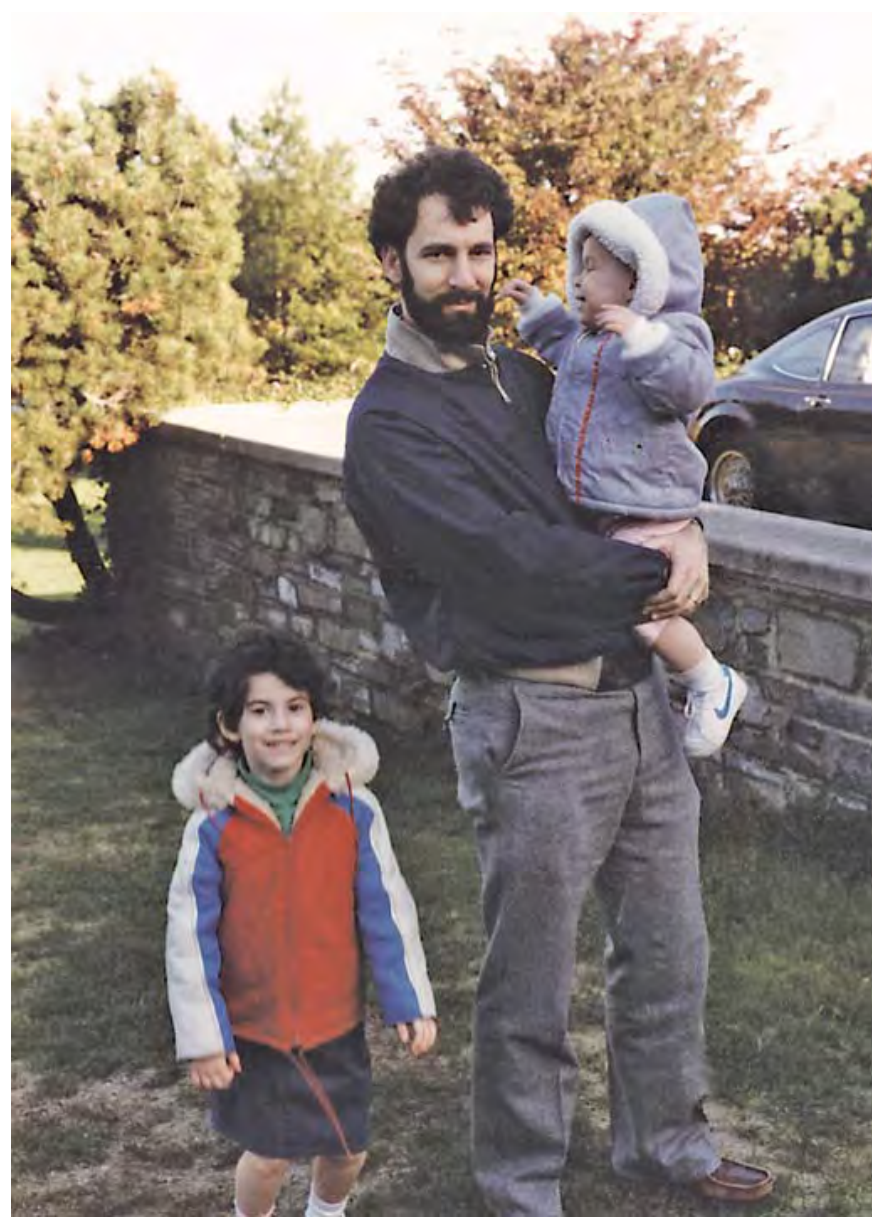

Charlie with daughters Nina (in red) and Lainie around 1983.

tied, but painting was never in the lead. Eventually he realized that he was much better at math. After knowing him for thirty-five years, if I had to account for his choice, I'd say that his passion for the beauty of math was what overtook everything else. I think that for him it's almost an addiction to the art of beautiful mathematics...Whenever I ask him to try to explain his work to me, his eyes sparkle and his voice and gestures are infused with an animation that is not present at any other time."

I met Charlie at the University of Chicago during the academic year 1971-1972. He was then a recently appointed full professor and I was a first-year graduate student. The Calderón-Zygmund seminar was probably the place where we first got acquainted. At the end of that academic year Charlie agreed to be my thesis advisor. Let me add that for me it was a fantastic experience; we are of the same age, and at that time we became close friends. We played ping-pong together in the Eckhard Hall basement and had long conversations about science, art, movies, music, politics, and, of course, mathematics.

I had the privilege of being his first graduate student, thereby initiating a set that now contains more than twenty elements. There is no doubt in my mind that the 


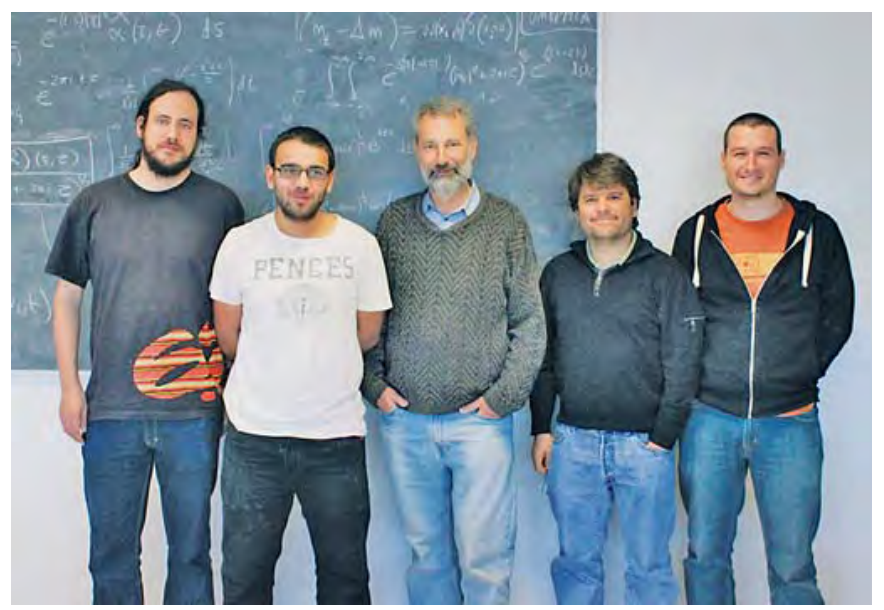

Charlie Fefferman with the ICMAT fluids team, Madrid circa 2011, from left to right: Javier Gómez-Serrano, Angel Castro, Charles Fefferman, Diego Córdoba, Francisco Gancedo

opportunity of enjoying Charlie's advice and friendship is an experience we all will treasure.

\section{Elias Stein}

\section{Fefferman's Early Work: The Epic Years (1969- 1974)}

I want to write about the first five years of Charlie Fefferman's major work, a brief period in which his many innovations transformed our views of several subjects in an intensive series of achievements unique in the history of modern mathematics.

\section{The Dissertation}

I first met Charlie in the fall of 1967 . He had started as a graduate student at Princeton the year before, but I was on leave that year. Our first real contact was after he had whizzed through his qualifying exam and said he wanted to write his thesis with me.

At that time the "Calderón-Zygmund paradigm," as it was later to be called, had already proved very successful in freeing parts of harmonic analysis from its heavy reliance on complex methods and leading it to the open vistas in higher dimensions, with new challenges and possibilities.

One question that particularly fascinated me was that of developing further methods for proving $L^{p}$ estimates, in particular where the critical limiting exponent for $p$ might be greater than 1, unlike the standard singular integrals. A problem of this kind arose in the Littlewood-Paley theory of square functions, and I proposed it to Charlie, eager to see what this promising eighteen-year-old could do.

To explain what was involved, there were first the more standard square functions, the $g$-function, and its

Elias Stein is emeritus professor of mathematics at Princeton University. His email address is stein@math. princeton. edu. nontangential version, the Lusin area integral $S$, for which it was known that the mappings $g \mapsto g(f), g \mapsto S(f)$ were of weak-type 1 and bounded on $L^{p}, 1<p<\infty$. Another more arcane ("tangential") variant arose in some problems, the function $g_{\lambda}^{*}$, defined by

(1)

$$
g_{\lambda}^{*}(f)(x)^{2}=\int_{\mathbb{R}_{+}^{n+1}}|\nabla u(x-y, t)|^{2}\left(\frac{t}{|y|+t}\right)^{n \lambda} t^{1-n} d y d t .
$$

Here $u(x, t)$ is the Poisson integral of a function $f$ on $\mathbb{R}^{n}$. In fact $g_{\lambda}^{*}$ dominated both $S$ and $g$, and it could be proved that $f \rightarrow g_{\lambda}^{*}(f)$ was bounded on $L^{p}$ if $1<\lambda<2$ and $p>2 / \lambda$; moreover, this failed when $p=2 / \lambda$. It seemed that the right assertion was that $f \mapsto g_{\lambda}^{*}(f)$ was of weak-type $p$ if $p>1$, but I had no real idea how to proceed, and this clearly required a new approach.

In just a short few weeks Charlie came back with the proof. I was surprised not only by the speed with which he had accomplished it but by the strength of his mathematics. I will not describe his idea of the proof but only say that it provided him a guide to solving the next problem-one that I proposed to him soon thereafter.

This problem concerned highly oscillating singular integrals. An example was the transformation $T: f \rightarrow f * K$ where the kernel $K$ (a distribution) is given by

$$
K(x)=\frac{e^{\frac{i}{|x|}}}{|x|^{n}} \text {, when } 0<|x| \leq 1
$$

and vanishes for $|x|>1$. It was known that the resulting $T$ was bounded on $L^{p}, 1<p<\infty$, but the hoped-for weak-type 1 result seemed out of reach.

Charlie set himself to work on this, and again, within a short few weeks he proved the desired assertion. His ideas were as follows.

To begin with he found the right restatement of the problem in its general form. It concerned a distribution kernel $K$ of compact support (represented by a function $K(x)$, when $x \neq 0$ ) which for a fixed parameter $\theta$, with $0 \leq \theta<1$, satisfied the following two conditions:
(i) $\hat{K}(\xi)=O\left(|\xi|^{-\theta \frac{n}{2}}\right)$ as $|\xi| \rightarrow \infty$,
(ii) $\int_{|x| \approx|y|^{1-\theta}}|K(x-y)-K(x)| d x \leq A$.

The standard Calderón-Zygmund kernels correspond to the case $\theta=0$, while the highly oscillatory ones correspond to $0<\theta<1$, with $\theta=\frac{1}{2}$ for the particular case (2) above.

With this incisive first step, he next decomposed an arbitrary $f$ in $L^{1}$, for fixed $\alpha>0$, in the standard way, writing $f=g+\sum_{j} b_{j}$, with $g$ in $L^{2},\|g\|_{L^{2}} \leq \alpha$, and the $b_{j}$ supported on disjoint cubes, while

$$
\frac{1}{\left|Q_{j}\right|} \int_{Q_{j}}\left|b_{j}\right| d x \approx \alpha \text { and } \int_{Q_{j}} b_{j} d x=0 .
$$

So $T(g)$ could be disposed with by the $L^{2}$ theory. Coming to the bad part, we let $B_{j}$ be the ball surrounding $Q_{j}$ (having the same center but twice the diameter). Here the main idea was to introduce the balls $B_{j}^{*}$, with the same center, but with

$$
\operatorname{diam} B_{j}^{*} \approx\left(\operatorname{diam} B_{j}\right)^{1-\theta} .
$$




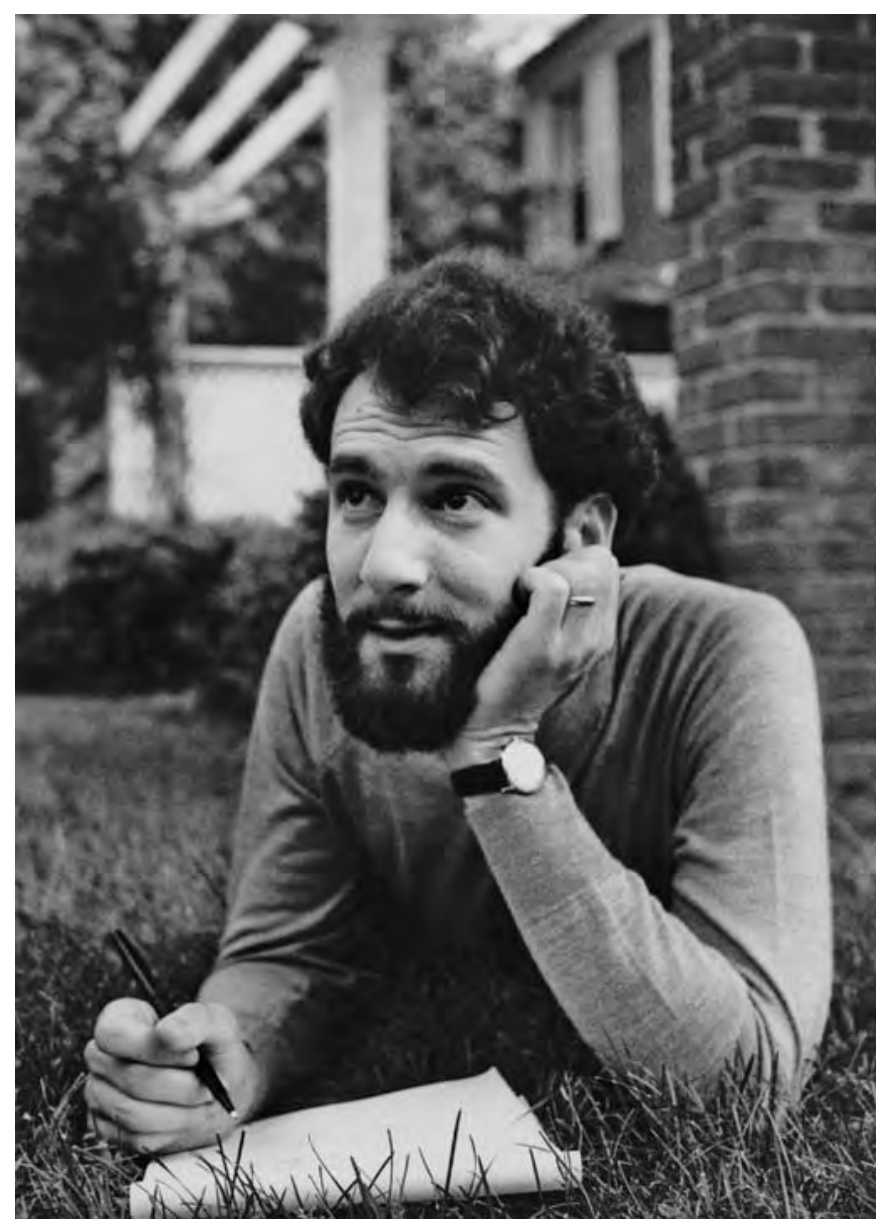

Charlie circa 1976 at Princeton.

(Only balls with diam $B_{j} \leq 1$ are significant here.) The contribution of $T\left(b_{j}\right)$ outside $B_{j}^{*}$ can be handled by (ii), as in the standard situation.

There remain the critical contribution of $T\left(b_{j}\right)$ in $B_{j}^{*} / B_{j}$ and the corresponding estimates of $T(\tilde{b})=\sum T\left(\tilde{b}_{j}\right)$ (where $\tilde{b}_{j}$ is a cleverly chosen replacement of $b_{j}$ ). At this stage he had prepared everything to go back to $L^{2}$ estimates, and he succeeded because he was able to combine directly two facts in this setting, namely,

$$
\begin{gathered}
\|T(\tilde{b})\|_{L^{2}} \lesssim\left\|(1-\Delta)^{-n \frac{\theta}{4}} \tilde{b}\right\|_{L^{2}} \text { and } \\
\left\|(1-\Delta)^{-n \frac{\theta}{4}} \tilde{b}\right\|_{L^{2}} \lesssim \alpha\|b\|_{L^{1}} .
\end{gathered}
$$

The result in its elegance and power, achieved so quickly, amazed me, as it would have anyone. Later his approach became a subject of much wider interest, as it was adapted to various other problems by S. Chanillo, M. Christ, Rubio de Francia, and others.

Because of these two striking successes, I had little hesitation in urging Charlie to attack a major problem, one which had concerned me for a number of years - that of the Bochner-Riesz means. To give some background:
In 1936 S. Bochner had introduced the operators $B_{R}^{\delta}$ defined by

$$
B_{R}^{\delta}(\hat{f})(\xi)= \begin{cases}\left(1-\frac{|\xi|^{2}}{R^{2}}\right)^{\delta} \hat{f}(\xi) & \text { for }|\xi|<R \\ 0 & \text { for }|\xi| \geq R\end{cases}
$$

These were later dubbed the Bochner-Riesz means (of order $\delta$ ). For $n=1, \delta=0$, these were the classical partial-sum operators that were closely related to the Hilbert transform, and thus one had $L^{p}$-norm control for $1<p<\infty$. Bochner had pointed out that for $n>1$, the order $\delta=\frac{n-1}{2}$ was the critical index in the sense that if $\delta>\frac{n-1}{2}$, then $B_{R}(f)=f * K_{R}$, where the kernels $K_{R}$ are good approximations to the identity, while for $\delta \leq \frac{n-1}{2}$ things are quite different and depend critically on the oscillatory nature of the $K_{R}$. By 1960 it was known that for each $p, 1<p<\infty$, one had $L^{p}$ control for some $\delta=\delta(p)<\frac{n-1}{2}$ (also convergence a.e. as $R \rightarrow \infty$ ), but these conclusions were far from optimal. In fact, one was led to expect that the $B_{R}^{\delta}$ when $\delta=0$ were bounded in $L^{p}$ for $\frac{2 n}{n+1}<p<\frac{2 n}{n-1}$ (in two dimensions $\left.\frac{4}{3}<p<4\right)$, together with the wider expectation that the $B_{R}^{\delta}$ were bounded outside that range when $\delta>n\left|\frac{1}{p}-\frac{1}{2}\right|-\frac{1}{2}$. Charlie's challenge was to go decisively beyond what was then known in a quest to achieve these ultimate goals.

In urging him to undertake this clearly difficult and uncertain effort, I could not be of much help, except for one thing I had observed the year before: when $n>1$ and $p$ is sufficiently close to 1 , the Fourier transform of an $L^{p}$ function can be restricted to the unit sphere $S^{n-1}$ of $\mathbb{R}^{n}$. More precisely, one had, in the present day terminology, the $\left(L^{p}, L^{q}\right)$ restriction phenomenon

$$
\left(\int_{S^{n-1}}|\hat{f}(\xi)|^{q} d \sigma(\xi)\right)^{\frac{1}{q}} \lesssim\|f\|_{L^{p}\left(\mathbb{R}^{n}\right)}
$$

when $q=2$, for $p$ close to 1 . The obvious suggestion was to explore the possible implications of this to the BR means.

Here Charlie succeeded marvelously with a remarkable conclusion: whenever the $\left(L^{p}, L^{2}\right)$ restriction phenomenon held, the optimal bounds for $B_{R}^{\delta}$ with the same $p$ would follow as a consequence.

After this accomplishment, we had some brief discussions on trying to extend the restriction phenomenon. By dualizing the problem we succeeded in obtaining the optimal conclusion in two dimensions: the $\left(L^{p}, L^{q}\right)$ restriction holds for $p=\frac{4}{3}-\epsilon$, and $q=\frac{4}{3}+\epsilon^{\prime}$, more precisely for $3 q=p^{\prime}$, with $1 \leq p<\frac{4}{3}$. All the above results are in Charlie's dissertation, published in 1970.

Shortly thereafter, two exciting developments followed. First, L. Carleson and P. Sjölin showed that in two dimensions, whenever $\delta>0$, one had boundedness of BR means for $\frac{4}{3} \leq p \leq 4$. This they did in part by adapting the philosophy for the restriction theorem in two dimensions. However gratifying this result was, it left open the pressing question: how to obtain the $L^{p}$ boundedness for the BR means of order $\delta=0$ in the range $\frac{4}{3}<p<4$. In other words, how to deal with the disc multiplier. 


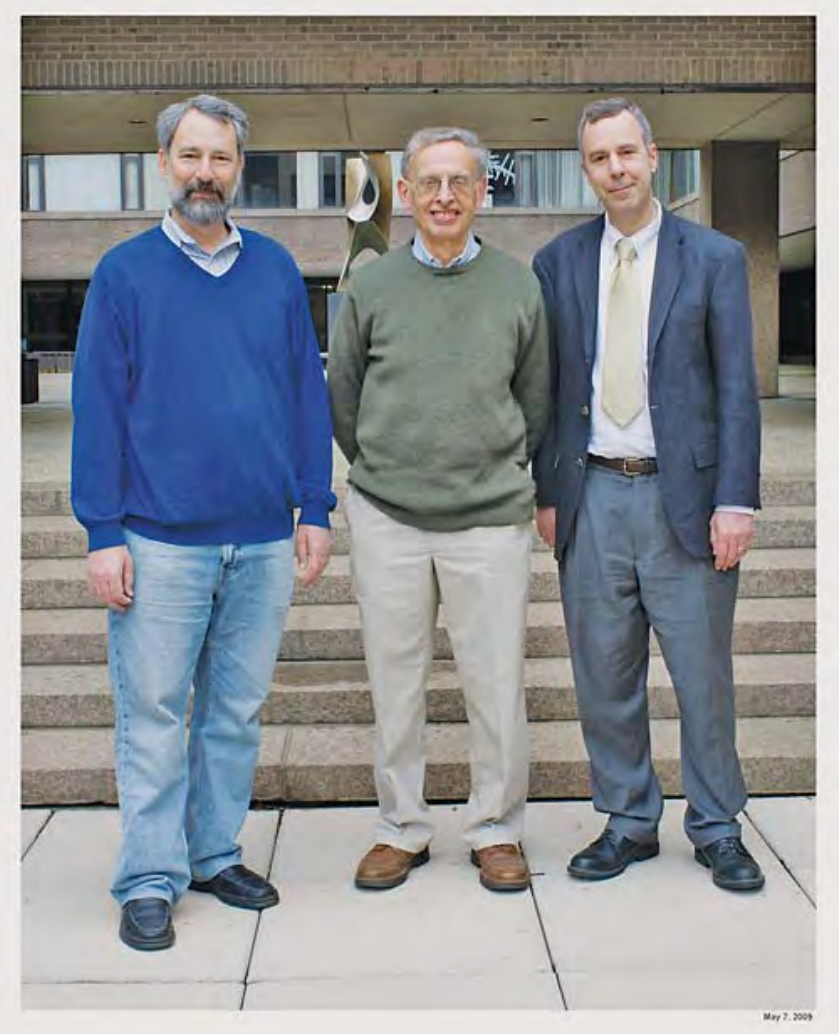

Eli Stein (center) with the Fefferman brothers at the conference to celebrate Stein's sixtieth birthday at Princeton in 1991. Charlie is on the left, Robert on the right.

Charlie's answer was an unexpected shock. His remarkable counterexample showed that the hoped-for result fails, except for the obvious case $p=2$. It dramatically transformed our view of the subject. This development and a number of Charlie's other achievements are covered in Terry Tao's section, so I will not say more about this direction of his work.

\section{The $H^{1}$-BMO Duality}

In the fall of 1970, after spending his first postdoctoral year in Princeton, Charlie took up a position at the University of Chicago. There he met Antoni Zygmund, who almost immediately put a question to him: what is the Poisson integral characterization of BMO? This question led Charlie to think more about BMO and ultimately to formulate and prove the famous aforementioned duality. (A charming reminiscence of his thinking about the issues involved can be found in his contribution to the collection All That Math: Portraits of Mathematicians as Young Readers.)

Let me briefly recall some facts about $H^{1}$ and BMO. The classical Hardy space $H^{p}, p>0$, arose at the intersection of complex analysis and Fourier analysis. Rephrased in the setting of the upper half-plane $\mathbb{R}_{+}^{2}$ with its boundary
$\mathbb{R}$, it consisted of those functions $F$ on $\mathbb{R}$ that arose as boundary values of the holomorphic functions $F$ in $\mathbb{R}_{+}^{2}$ that are uniformly bounded in $L^{p}$ on all lines in $\mathbb{R}_{+}^{2}$ parallel to $\mathbb{R}$. Formally, each such $F$ can be represented as $F=f+i H(f)$, where $f$ is real-valued and $H$ is the Hilbert transform. Thus if $1<p<\infty$, classical $H^{p}$ was essentially real $L^{p}(\mathbb{R})$. However, when $p \leq 1$, things were quite different, and $H^{p}$ had all sorts of interesting properties either not valid for $L^{1}$ or totally missing for the trivial space $L^{p}$ when $p<1$.

By 1960 it was clearly time to see whether there was anything like real Hardy spaces in $\mathbb{R}^{n}$, for $n>1$. Here G. Weiss and I had some initial success. We defined the space $H^{1}\left(\mathbb{R}^{n}\right)$ to consist of those $L^{1}$ functions for which, in addition, their Riesz transforms, $R_{j}(f), j=1, \ldots, n$, also belonged to $L^{1}$. It turned out that this space had a number of interesting features going beyond $L^{1}$, in particular a maximal characterization involving Poisson integrals in terms of $L^{1}$ (and not merely weak- $L^{1}$ ). A further hint that $H^{1}\left(\mathbb{R}^{n}\right)$ was the appropriate substitute for $L^{1}\left(\mathbb{R}^{u}\right)$ came several years later, when it was seen that classical singular integrals, broadly speaking, took $H^{1}\left(\mathbb{R}^{n}\right)$ to $L^{1}\left(\mathbb{R}^{n}\right)$ and in fact preserved $H^{1}\left(\mathbb{R}^{n}\right)$.

Let us come to the other branch of the tree: BMO. That space (functions of bounded mean oscillation) appeared first in 1961 in the work of F. John and L. Nirenberg. A function $f$ on $\mathbb{R}^{n}$ was said to be in BMO if

$$
\sup _{Q} \frac{1}{|Q|} \int_{Q}\left|f-f_{Q}\right| d x \leq A<\infty .
$$

Here $f_{Q}=\frac{1}{|Q|} \int_{Q} f d x$, and the $Q$ range over all cubes $Q$ in $\mathbb{R}^{n}$.

John and Nirenberg proved the remarkable fact that (4) implies an analogous inequality with $\left|f-f_{Q}\right|$ replaced by $\left|f-f_{Q}\right|^{p}$, for any $p<\infty$, and in fact replaced by $e^{c\left|f-f_{Q}\right|}$ for an appropriate positive $c$.

This kind of result had immediate consequences: in the work of John for rotation and strain of mappings and in J. Moser's elegant proof of the DiGiorgi-Nash estimates. Then in 1966 the relevance of BMO to harmonic analysisand the possibility that it might serve as the appropriate substitute for $L^{\infty}$-became a little clearer when J. Peetre, S. Spanne, and I (quite independently) observed that the standard singular integrals took $L^{\infty}$ to BMO and, even better, that BMO was stable under these transformations.

With this we return to the question Zygmund asked Charlie: to characterize BMO by Poisson integrals. Charlie's answer was given in terms of three interrelated assertions, each remarkable in its own right:

(i) The dual space of $H^{1}$ is BMO.

(ii) A function $f$ is in BMO if and only if

$f=f_{0}+\sum_{j=1}^{n} R_{j}\left(f_{j}\right)$, where $f_{0}, f_{1}, \ldots, f_{n}$ are all in $L^{\infty}$.

(iii) $f$ is in BMO if and only if $t|\nabla u(x, t)|^{2} d x d t$ is a Carleson measure. 
The assertion (iii) was the answer Zygmund sought but did not expect! Here $u(x, t), x \in \mathbb{R}^{n}, t>0$, is the Poisson integral of $f$, and the statement (iii) was that

$$
\sup _{B} \frac{1}{|B|} \int_{T(B)} t|\nabla u(x, t)|^{2} d x d t \leq A<\infty,
$$

where $T(B)=\{x, t:|x-y|<r-t, 0<t<r\}$ is the "tent" over the ball $B \subset \mathbb{R}^{n}$, centered at $y$ of radius $r$, and $B$ ranges over all balls. It is noteworthy that measures of this kind arose for $n=1$ in Carleson's work in 1962 on the corona conjecture.

Charlie's discovery reawakened interest in Hardy spaces and BMO, and a flurry of activity followed immediately. Working together we developed a systematic theory of Hardy spaces, which in particular freed it from its reliance on harmonic functions. This showed that an element of $H^{p}$ could be characterized in a variety of different but equivalent ways:

(i) in terms of maximal characterizations by general smooth approximations of the identity (not just restricted to Poisson integrals),

(ii) similarly, in terms of general square functions, and

(iii) in terms of Riesz transforms and their higher analogues.

A consequence of this was the confirmation of the status of $H^{1}$ as the rightful substitute for $L^{1}$ (and $H^{p}$ for $L^{p}, p<1$ ) in the theory of singular integrals; not only was it stable under the action of these operators, but appropriate maximal and square functions had $L^{1}$ control, and there was also a useful Calderón-Zygmund decomposition in this context.

Another interesting by-product was the function $f^{\#}$ defined by

$$
f^{\#}(x)=\sup _{x \in Q} \frac{1}{|Q|} \int_{Q}\left|f-f_{Q}\right| d x .
$$

In analogy with the John-Nirenberg theorem one had that $f^{\#} \in L^{p}$ implied that $f \in L^{p}$, if $p<\infty$, and this was useful in resolving the problem of complex interpolation between $H^{1}$ or BMO and $L^{p}, 1<p<\infty$.

A significant further development started with Charlie's observation that the duality of $H^{1}$ and BMO can be restated by saying each $f \in H^{1}$ has an atomic decomposition. This meant that any such $f$ could be written as $\sum_{j} \lambda_{j} a_{j}$, where each atom, $a_{j}$, was supported on a cube $Q_{j}$, with $\left|a_{j}(x)\right| \leq \frac{1}{\left|Q_{j}\right|}, \int a_{j}(x) d x=0$, and the constants $\lambda_{j}$ satisfied $\sum_{j}\left|\lambda_{j}\right|<\infty$.

In the hands of R. Coifman, C. Herz, R. Latter, G. Weiss, and others, the idea of atomic decomposition was greatly developed, encompassing $H^{p}, p<1$, and ultimately becoming the preferred starting point for various extensions of the theory.

\section{The Mapping Theorem and Bergman Kernel}

In the following years Charlie made a number of other notable advances. Any list would have to include his version of Carleson's theorem about the convergence of Fourier series, his solution with R. Beals of an outstanding local solvability problem for linear partial differential equations, and his work involving the mapping theorem and the Bergman kernel-to which I'll limit myself.

While still at the University of Chicago, Charlie was told by R. Narasimhan of an outstanding problem in several complex variables: Suppose $\Omega_{1}$ and $\Omega_{2}$ are a pair of bounded domains in $\mathbb{C}^{n}, n>1$, each having a smooth $\left(C^{\infty}\right)$ boundary and each being strongly pseudoconvex. Suppose there is a bijective biholomorphism $\Phi$ from $\Omega_{1}$ to $\Omega_{2}$. Then does $\Phi$ extend to a smooth diffeomorphism of $\bar{\Omega}_{1}$ to $\bar{\Omega}_{2}$ ?

It must be stressed that the case $n \geq 2$ differs essentially from the classical case $n=1$. First, there is no adequate theory of conformal mappings to help when $n \geq 2$. In particular, two domains may be such that $\bar{\Omega}_{1}$ and $\bar{\Omega}_{2}$ are close (in $C^{\infty}$ ) without there existing a biholomorphism between them. Moreover, pseudoconvexity must enter the picture, one way or another. After explaining the problem, Narasimhan said to Charlie, "You must do that."

Charlie was immediately ... as inspired and natural in conception as it was difficult and painful in execution...

remembered a course he attended as a graduate student on boundary behavior of holomorphic functions and having been intrigued by the Bergman kernel and resulting metric. The facts about these are as follows.

The Bergman kernel $K_{\Omega}(z, w)$ of domain $\Omega$ is determined by the fact that $f \mapsto \int_{\Omega} K_{\Omega}(z, w) f(w) d w$ is the orthogonal projection of $L^{2}(\Omega, d w)$ onto the subspace of $L^{2}$ holomorphic functions on $\Omega$. A remarkable feature was that if the Hermitian (Riemannian) metric $d s^{2}=\sum \frac{\partial}{\partial \bar{z}_{j} \partial z_{k}}\left(\log K_{\Omega}(z, z)\right) d \bar{z}_{j}, d z_{k}$ is attached to $\Omega$, then a biholomorphism $\Phi: \Omega_{1} \rightarrow \Omega_{2}$ induces an isometry in these metrics.

Charlie's idea was as inspired and natural in conception as it was difficult and painful in execution. In brief, what had to be done was to carry out the following plan: after fixing corresponding points $P_{1} \in \Omega_{1}, P_{2}=\Phi\left(P_{1}\right)$, follow any geodesic on $\Omega_{1}$ starting at $P_{1}$ and the corresponding geodesic $\Omega_{2}$ to infinite time when both reach the boundary at points $Q_{1} \in \partial \Omega_{1}$ and $Q_{2} \in \partial \Omega_{2}$. The mapping $Q_{1} \mapsto Q_{2}$ should then yield the correspondence of the boundaries and thus give the $C^{\infty}$ extension of $\Phi$.

The first step therefore was analyzing the behavior of the Bergman metric, which required a clearer understanding of the Bergman kernel, a question of great interest in its own right. The description of $K_{\Omega}$ he obtained was as follows. Assuming that $\Omega$ is a bounded domain with $C^{\infty}$ boundary which is strongly pseudoconvex, then

$$
K_{\Omega}(z, w)=\frac{A(z, w)}{(Q(z, w))^{n+1}}+B(z, w) \log Q(z, w) .
$$

Here $Q(z, w)$ is the holomorphic part of the secondorder Taylor expansion of a defining function $\rho$ of the domain $\Omega ; A$ and $B$ are smooth functions, with 


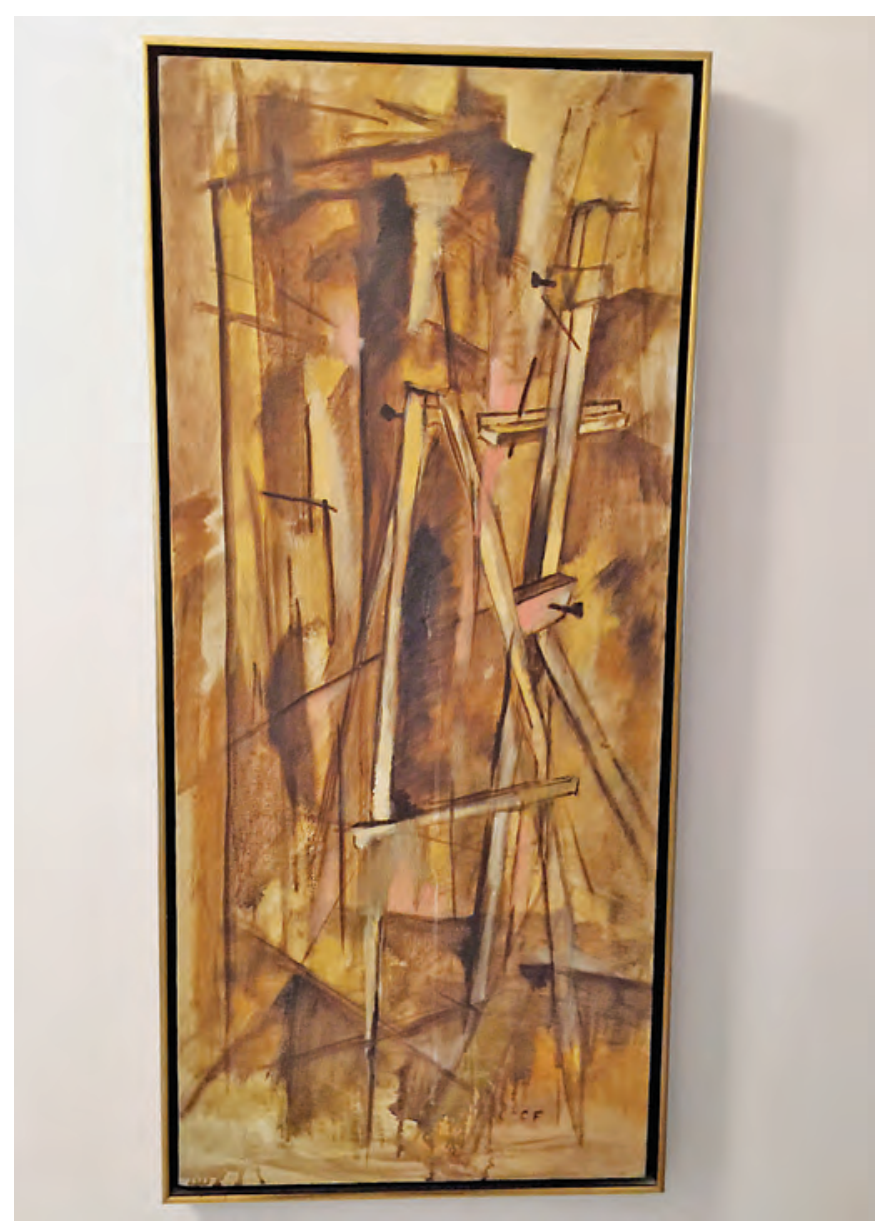

Studio Interior, painted by Charlie circa 1968.

$A(z, z) \neq 0$. It should be noted that in the case $\Omega$ is the unit ball, we can take $\rho(z)=1-|z|^{2}, Q(z, w)=1-z \bar{w}$, and $A(z, w) \equiv c_{n}, B(z, w) \equiv 0$. Moreover, it should be stressed that no such general result has been proved (or even formulated!) if one drops the assumption of strong pseudoconvexity.

To prove (5) required that at each boundary point $w \in \partial \Omega$ one osculate to a high degree a version of a complex ball and transplant its explicit Bergman kernel to $\Omega$. This gave a first-order approximation to $K_{\Omega}$, which then had to be followed by a highly intricate iterative procedure to ultimately obtain (5).

Once one has (5) and the Bergman metric is controlled, one can proceed to the main lemma: suppose $X\left(t, P_{0}, \xi\right)$ is the point of the geodesic starting at $P_{0}$ in the unit direction $\xi$ and at time $t$. Assume that for some $\xi_{0}$ the nonnegative time portion on the geodesic $X\left(t, P_{o}, \xi_{0}\right)$ does not lie in a compact set. Then

(i) $\lim _{t \rightarrow \infty} X\left(t, P_{0}, \xi_{0}\right)$ converges to a boundary point of $\Omega$.

(ii) The same is true for all $\xi$ near $\xi_{0}$, and the resulting mapping of $\xi \mapsto X\left(\infty, P_{0}, \xi\right)$ is a local diffeomorphism.

(iii) All boundary points can be reached this way.
The proof of the lemma requires again a highly involved argument, because, among other things, it is imperative to overcome the obstacle of the log term in (5). When finally all this is done the theorem is:

Let $\Omega_{1}$ and $\Omega_{2}$ be a pair of bounded domains with $C^{\infty}$ boundaries that are strongly pseudoconvex. Suppose there is a biholomorphism between them. Then this extends to a $C^{\infty}$ diffeomorphism between $\bar{\Omega}_{1}$ and $\bar{\Omega}_{2}$.

After his paper (1974) various alternate approaches, simplifications, or extensions were obtained by others, including: L. Boutet de Monvel and J. Sjöstrand (1976); S. Bell and E. Ligocka (1980); L. Nirenberg, S. Webster, and P. Yang (1980); S. Bell and D. Catlin (1982); and F. Forsternic (1992). Still, more than forty years later, Charlie's achievement stands as a milestone in the development of several complex variables.

\section{Terence Tao}

\section{Connecting Fourier Analysis and Geometry}

During the early 1970s Fefferman made a number of important and fundamental contributions in the theory of oscillatory singular integrals, the study of which can be motivated by the classical problem of determining the nature of convergence of Fourier series and Fourier integrals and which has since had remarkable connections and applications to many other fields of mathematics, including partial differential equations, analytic number theory, and geometric measure theory.

To motivate the subject, let us first work in the simplest setting of Fourier series on the unit circle $\mathbb{R} / \mathbb{Z}$. Given any absolutely integrable function $f: \mathbb{R} / \mathbb{Z} \rightarrow \mathbb{C}$, one can form the Fourier coefficients $\hat{f}(n)$ for any integer $n$ by the formula

$$
\hat{f}(n):=\int_{\mathbb{R} / \mathbb{Z}} f(x) e^{-2 \pi i n x} d x,
$$

and then the Fourier inversion formula asserts that one should have the identity

$$
f(x)=\sum_{n=-\infty}^{\infty} \hat{f}(n) e^{2 \pi i n x} .
$$

If $f$ is sufficiently regular, then there is no difficulty in interpreting and proving this identity; for instance, if $f$ is continuously twice differentiable, one can show that the Fourier coefficients $\hat{f}$ are absolutely summable and that the series appearing on the right-hand side of (6) converges uniformly to $f$. If $f$ is instead assumed to be square-integrable, then the Fourier coefficients need not be absolutely summable any more, but it follows easily from Plancherel's theorem that the series in (6) still converges unconditionally to $f$ within the Hilbert space $L^{2}(\mathbb{R} / \mathbb{Z})$.

The situation becomes more subtle if one weakens the regularity hypotheses on $f$ or asks for stronger notions of convergence. Suppose for instance that $f$ is an arbitrary $p^{\text {th }}$-power integrable function for some $1<p<\infty$ not

Terence Tao is professor of mathematics at the University of California, Los Angeles. His email address is tao@math . ucla . edu. 
equal to 2 ; thus $f \in L^{p}(\mathbb{R} / \mathbb{Z})$. As it turns out, the series on the right-hand side of (6) will not, in general, be unconditionally convergent or absolutely convergent in $L^{p}(\mathbb{R} / \mathbb{Z})$; however, the partial sums

$$
S_{N}(f)(x):=\sum_{n=-N}^{N} \hat{f}(n) e^{2 \pi i n x}
$$

will still converge in $L^{p}(\mathbb{R} / \mathbb{Z})$ norm to $f$. Establishing this fact is equivalent (by the uniform boundedness principle) to demonstrating that operators $S_{N}$ are uniformly bounded in $L^{p}(\mathbb{R} / \mathbb{Z})$. The operators $S_{N}$ can be explicitly described as an integral operator:

$$
S_{N} f(x)=\int_{\mathbb{R} / \mathbb{Z}} f(x-t) \frac{\sin \left(\left(N+\frac{1}{2}\right) t\right)}{\sin (t / 2)} d t .
$$

The kernel $\frac{\sin \left(\left(N+\frac{1}{2}\right) t\right)}{\sin (t / 2)}$ has a numerator which oscillates rapidly when $N$ is large and a denominator that goes to zero as $t$ goes to zero. As such it is a simple example of an oscillatory singular integral operator (though in this case, it is technically not singular because the numerator also vanishes at $t=0$, though its derivative is quite large at that point). There are by now many techniques to establish the required boundedness for $1<p<\infty$; for instance, one can use the Calderón-Zygmund theory of singular integral operators. On the other hand, boundedness (and hence convergence) in $L^{p}$ norm is known to fail when $p=1$ or $p=\infty$.

A more difficult question is whether the partial sums $S_{N}(f)$ converge pointwise almost everywhere to $f$. (One can construct examples to show that pointwise everywhere convergence can fail, even if $f$ is assumed to be continuous.) This turns out to basically be equivalent to establishing the boundedness (or more precisely, weak-type boundedness) in $L^{p}$ of not just each individual operator $S_{N}$, but the more complicated Carleson maximal operator

$$
C f(x):=\sup _{N>0}\left|S_{N}(f)(x)\right| .
$$

Controlling this operator is substantially more difficult than controlling a single $S_{N}$, but this was famously achieved in 1966 by Lennart Carleson (for $p=2$ ) and then by Richard Hunt (for general $1<p<\infty$ ). On the other hand, a famous construction of Kolmogorov produces an absolutely integrable function $f$ whose partial Fourier series $S_{N}(f)$ diverges pointwise almost everywhere (or even everywhere, if one is more careful in the construction).

Fefferman made multiple contributions to these questions and their higher-dimensional analogues; we shall restrict our attention here to just two of his most wellknown and influential works in this area. Firstly, for higher-dimensional Fourier series, if the circle $\mathbb{R} / \mathbb{Z}$ is replaced by a torus $(\mathbb{R} / \mathbb{Z})^{d}$ for some $d \geq 2$, Fefferman studied the ball multiplier

$$
S_{N}(f)(x):=\sum_{n \in \mathbb{Z}^{d}:|n| \leq N} \hat{f}(n) e^{2 \pi i x \cdot n} d x,
$$

where $|n|$ denotes the Euclidean norm of $n$; thus $S_{N}$ sums the terms of the Fourier series whose frequency $n$ lies in the ball of radius $N$. On the one hand, Fefferman

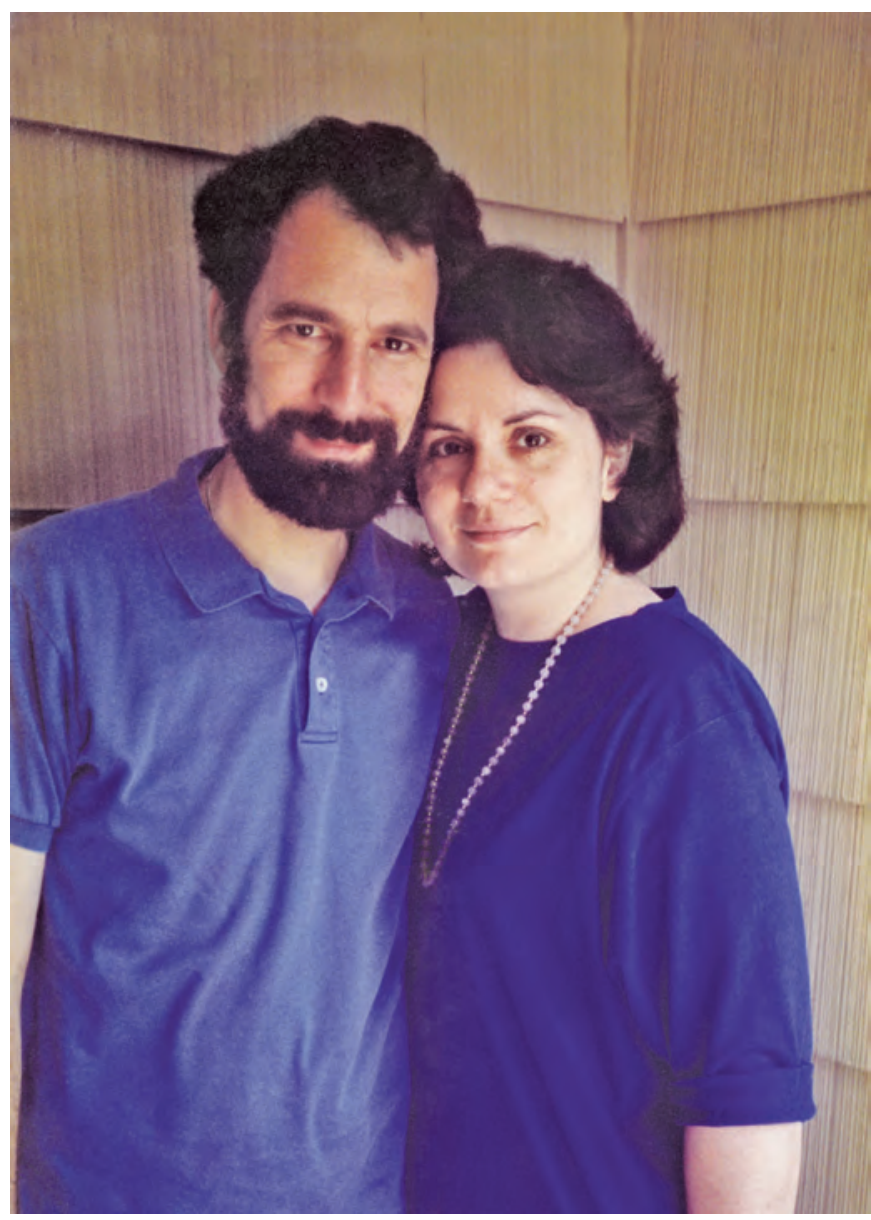

Charlie with his wife, Julie, in front of their home in Princeton circa 1980.

observed that if one replaced the ball with a cube, then a modification of the onedimensional theory allowed one to establish $L^{p}$ boundedness and convergence of these multipliers for all $1<p<\infty$. On the other hand, he showed the surprising fact that the ball multipliers were not bounded uniformly on $L^{p}$ for any $p \neq 2$, which implied in particular that one could construct functions in $L^{p}\left((\mathbb{R} / \mathbb{Z})^{d}\right)$ whose partial Fourier series $S_{N}(f)$ did not converge in $L^{p}$ norm! We can describe a modern version of Fefferman's remarkable construction here in the two-dimensional case $d=2$. This construction revealed an important connection between such Fourier-analytic problems and questions in 
geometric measure theory and in particular the Kakeya needle problem, which asked one to find the minimal area subset of the plane in which one could rotate a unit line segment (or "needle") by a full rotation. In 1919 Abram Besicovitch gave a construction that implied that such a rotation could be carried out in a set of arbitrarily small measure. A modification of this construction produces, for any given $\varepsilon>0$, a family of rectangles $R_{1}, \ldots, R_{n}$ which overlapped heavily in the sense that the measure of the union of the $R_{i}$ was less than $\epsilon$ times the sum of the individual measures, but such that if one translated each rectangle $R_{i}$ along its long axis by (say) three times its length, the resulting rectangles $\tilde{R}_{1}, \ldots, \tilde{R}_{n}$ were disjoint. On the other hand, after suitable rescaling of this family of rectangles and taking $N$ large enough, it is possible to construct a wave packet $f_{i}$ supported on each shifted rectangle $\tilde{R}_{i}$ such that the two-dimensional ball multiplier $S_{N}$, when applied to $f_{i}$, became large on the rectangle $R_{i}$. By testing $S_{N}$ on a suitable linear combination of these wave packets $f_{i}$, one can demonstrate the unboundedness of $S_{N}$ for any $p>2$, and a duality argument then handles the remaining case $p<2$.

The fundamental connection between higherdimensional Fourier analysis and Kakeya-type questions has guided much of the subsequent work in the area; it is now standard practice to control oscillatory integral operators (such as $S_{N}$ ) by first decomposing the functions involved into wave packets such as the functions $f_{i}$ mentioned above, apply the operators to each wave packet individually, and use a combination of Fourier analytic methods and geometric analysis to control the superposition of these operators. A recent triumph of these sets of techniques has been the resolution last year of the Vinogradov main conjecture in analytic number theory by Bourgain, Demeter, and Guth, which gives near-optimal bounds on the mean values of exponential

\section{Selected Honors and Distinctions of Charles Fefferman}

Raphael Salem Prize 1971

American Academy of Arts and Sciences 1972

Alan T. Waterman Award 1976

Fields Medal 1978

National Academy of Sciences 1979

Doctor Honoris causa: University of Maryland 1979

American Philosophical Society 1985

Doctor Honoris causa: Universidad Autónoma de Madrid 1990

Bergman Prize 1992

Bôcher Prize $2008^{a}$

Wolf Prize 2017

a See article in April 2008 Notices, www.ams.org/notices /200804/tx080400499p.pdf sums such as

$$
\sum_{n=1}^{N} e^{2 \pi i\left(\alpha_{1} n+\alpha_{2} n^{2}+\cdots+\alpha_{k} n^{k}\right)}
$$

as one varies the frequencies $\alpha_{1}, \ldots, \alpha_{k}$ and which has important consequences in analytic number theory.

In 1973 Fefferman revisited Carleson's theorem on the pointwise convergence of Fourier series and gave a striking new proof that was a key impetus for the modern field of time-frequency analysis. Whereas Carleson's argument focused on carefully decomposing the function $f$, Fefferman's strategy proceeded by instead decomposing the operator $C$. The first step was to linearize this operator by replacing it with the infinite family of operators

$$
\begin{aligned}
S_{N(\cdot)}(f)(x) & :=S_{N(x)} f(x) \\
& =\int_{\mathbb{R} / \mathbb{Z}} f(x-t) \frac{\sin \left(\left(N(x)+\frac{1}{2}\right) t\right)}{\sin (t / 2)} d t,
\end{aligned}
$$

where $N$ ranged over all measurable functions $N: \mathbb{R} / \mathbb{Z} \rightarrow$ $\mathbb{Z}$ from the circle to the integers. As no regularity hypotheses are placed on the function $N$, this integral operator is very rough and seemingly hopeless to attack by conventional harmonic analysis methods. However, guided by key examples of these functions $N$, Fefferman realized that the graph of the function $N$ (viewed as a subset of phase space $\mathbb{R} / \mathbb{Z} \times \mathbb{Z}$ ) could be used as a sort of road map to efficiently decompose the operator $S_{N(\cdot)}$ into components indexed by phase space rectangles (or tiles), which could then be organized into trees and then forests, the contributions of which could be estimated by a combination of clever combinatorial arguments and the almost orthogonality of various pieces of the operator. This highly original argument took some time to be properly integrated with the rest of the subject, but through the work of Michael Lacey, Christoph Thiele, and others, starting in the late 1990s, the techniques of Carleson and Fefferman were unified with more traditional tools from Calderón-Zygmund theory to obtain a systematic set of methods in time frequency analysis to control highly singular operators that had previously been out of reach, such as the bilinear Hilbert transform of Calderón, as well as many variants of relevance to ergodic theory or to scattering theory.

\section{Louis Nirenberg}

\section{Charlie Fefferman on PDEs}

As is well known, Charlie Fefferman was a child prodigy: when he was in fourth grade he read, and understood, math books going up to calculus. He received his $\mathrm{PhD}$ at Princeton at age twenty. His adviser was Eli Stein, also a wonderful mathematician and mentor.

I first met Charlie around 1972, shortly after he was made full professor at the University of Chicago at age twenty-two-the youngest full professor anywhere. I still remember listening with great pleasure to his invited

Louis Nirenberg is emeritus professor of mathematics at New York University. His email address is ni renberg@cims. nyu. edu. 


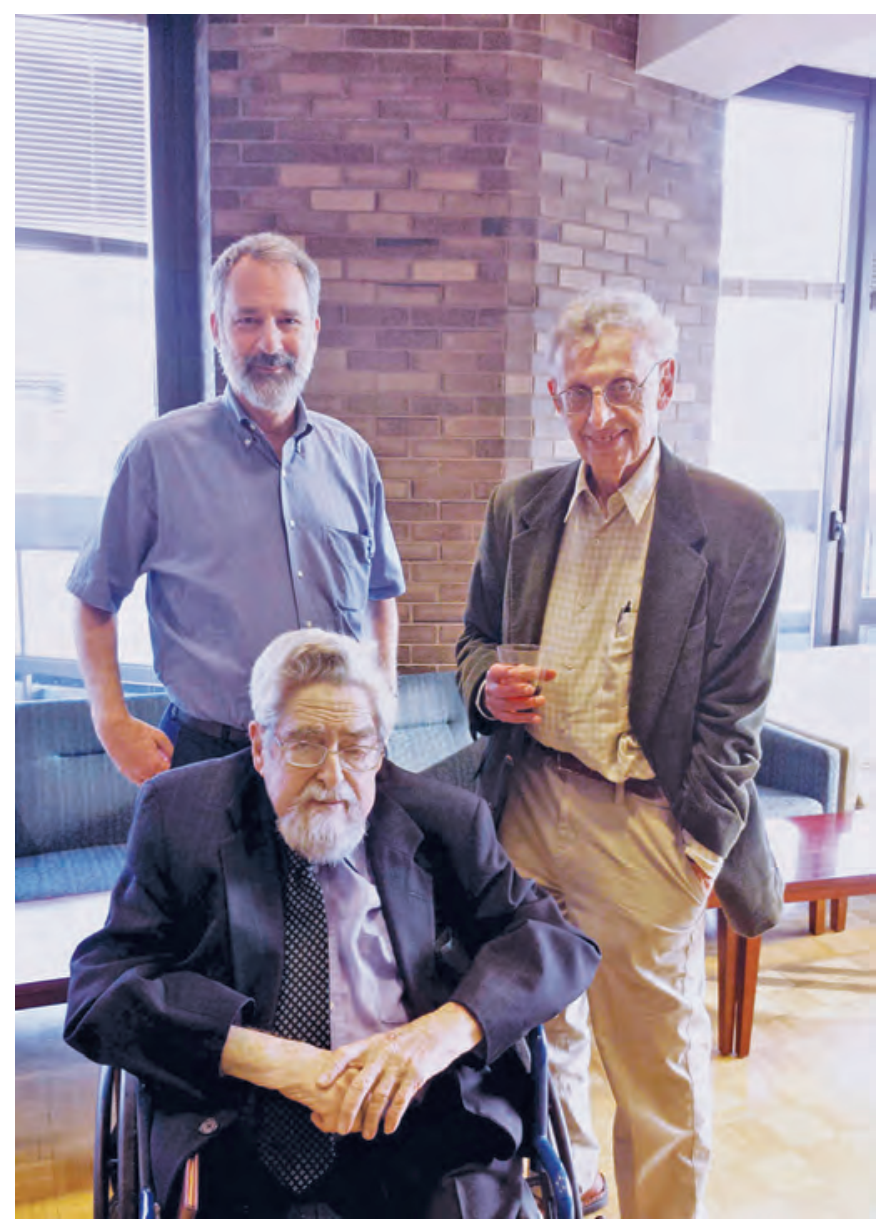

Louis Nirenberg (seated), Charlie, and Elias Stein at a celebration of Nirenberg's Abel Prize, Courant Institute, New York, circa 2015.

address at the International Congress in Vancouver in 1974 on recent progress in classical Fourier analysis.

Charlie has made fundamental contributions in an enormously wide range of subjects: harmonic analysishe's a world master; analysis on complex manifolds; microlocal analysis and linear partial differential (and pseudodifferential) operators; quantum mechanics; fluid flow, Euler, and Navier-Stokes equations; and generalizing Whitney's extension theorem.

I will confine myself to a few topics connected with partial differential equations and related things. In 1971 Charlie proved that the space of functions BMO is the dual to the Hardy space $H^{1}$.

F. Treves and I presented a condition, $P$, which we conjectured would be necessary and sufficient for local solvability of linear PDEs of principal type. We proved this in the case that the real and imaginary parts of the leading coefficients are real analytic. Shortly afterwards Charlie and R. Beals proved the sufficiency of $P$ in the nonanalytic case.

Charlie, in collaboration with A. Córdoba and D. H. Phong, wrote a series of deep papers connected with microlocal analysis and PDEs. In 1985 Charlie wrote a beautiful expository paper on "the uncertainty principle" describing some of their work. It contains a wealth of deep results; I recommend that all students studying PDE read it. If a function $u$ is mainly concentrated in a box $Q$ and its Fourier transform is concentrated in a box $Q^{\prime}$, one says that $u$ is microlocalized in $Q \# Q^{\prime}$. The uncertainty principle says, essentially, that $|Q| \cdot\left|Q^{\prime}\right| \geq 1$. They study more complicated regions $B_{a}$ (than the cubes) and decompose $L^{2}$ functions into a sum of components microlocalized using the $B_{a}$. The decomposition is used to diagonalize pseudodifferential operators modulo small errors. There are applications to solvability, to fundamental solutions, and to Schrödinger equations. Connections with Egorov's theorem and quantum theory are given. Using this a pseudodifferential operator is reduced to a multiplier. Their work also connects with symplectic geometry. It is simply striking.

Charlie has written many deep papers on mathematical physics. A long one, in 1986, ...why states of matter, such as molecules, form at suitable temperatures... studies the quantum mechanics of $N$ electrons and $M$ nuclei. He tries to understand why states of matter, such as molecules, form at suitable temperatures. He obtains very striking results, but the general problem is still open. Things depend on the lowest eigenvalue of a Hamiltonian. With L. Seco he wrote a series of interesting papers. They obtain asymptotic formulas for the ground state of a nonrelativistic atom. Very refined estimates for the eigenvalues and eigenfunctions of an ODE are obtained.

Charlie also wrote a number of interesting papers in fluid dynamics with various co-authors. With Donnelly he wrote a lovely paper on nodal sets for real eigenfunctions $F$ satisfying $\Delta F+a F=0$ on a compact connected Riemannian manifold. Near the nodal set $N$ (where $F$ vanishes) they prove that $F$ vanishes on $N$ to at most order $c|a|^{1 / 2}$. The paper contains a variety of results, including behavior near zeros of holomorphic functions in higher dimensions.

I would like to add that I consider Charlie one of the deepest and most brilliant mathematicians I have ever met. He is also an excellent speaker, and his papers are a pleasure to read. He and his wife, Julie, have been close friends all these years. 


\section{Joseph J. Kohn}

\section{Fefferman's Contributions to the Theory of Several Complex Variables}

Charles L. Fefferman has made numerous fundamental contributions to the theory of several complex variables (SCV). His published papers have had a major impact on the field. His expository writing, his lectures, and his discussions have provided students, colleagues, and collaborators with much inspiration and deep insights into the subject. He obtained many seminal results in SCV covering a large range of topics; it is not possible to give a coherent description of all these within the limits of this article. Here I will briefly describe some of the highlights of his groundbreaking work.

Fefferman's first contribution to complex analysis was a remarkable achievement: He proved boundary regularity of holomorphic mappings using the Bergman metric. His proof is highly original, it is a tour-de-force. It is described in more detail by Stein above.

One of the principal themes in SCV is the study of domains of holomorphy in $\mathbb{C}^{n}$. Let $D \subset \mathbb{C}^{n}$ be a domain with smooth boundary $\partial D$. Then it is a domain of holomorphy if and only if it is pseudoconvex, which means that the Levi form is nonnegative. Great progress has been made in the study of the analysis and geometry associated with strictly pseudoconvex domains (that is, when the Levi form is positive definite), much of it due to Fefferman's groundbreaking research. In "The Bergman kernel and biholomorphic mappings of pseudoconvex domains," Fefferman proves a fundamental result: if $F: D_{1} \rightarrow D_{2}$ is a biholomorphic map between two strictly pseudoconvex domains, then it is smooth up to the boundary of $D_{1}$. This result is a generalization of the Riemann mapping theorem; it enables one to attach invariants to the boundary points, providing a powerful hold on the classification problem. Fefferman's proof of this result involves a profound analysis of the Bergman kernel and metric; it is sketched in Stein's section.

Fefferman continues his study of strictly pseudoconvex domains in "Monge-Ampère equations, the Bergman kernel, and geometry of pseudoconvex domains." Here he studies their geometry, in particular the behavior of chains-curves on the boundary that are preserved by biholomorphic maps; they are analogous to geodesics in Riemannian geometry. To compute the Bergman metric and its associated Hamiltonian, Fefferman considers the Dirichlet problem for the following Monge-Ampère equation:

$$
J(u)=(-1)^{n} \operatorname{det}\left(\begin{array}{cc}
u & u_{\bar{k}} \\
u_{j} & u_{j \bar{k}}
\end{array}\right)_{i \leq j, k \leq n}=1
$$

in $D$ with $u=0$ on $\partial D$. Then the Bergman kernel is replaced by $C_{n}(u(z))^{-(n+1)}$ with $u$ as above. The difficulty here is that it is not known whether such a $u$ exists. Nevertheless, Fefferman finds a method of finding

Joseph J. Kohn is emeritus professor of mathematics at Princeton University. His email address is kohn@math. princeton . edu. formal approximate solutions, and with these, using the smoothness of biholomorphic mappings, he actually computes the metric $d s^{2}$ and its associated Hamiltonian. He uses this to show some surprising behavior of chains.

Fefferman's study of these problems continues in the monumental paper "Parabolic invariant theory in complex analysis." His analysis starts with an analogy between Riemannian manifolds $M$ and strictly pseudoconvex domains $D$. An isometry of $M$ in normal coordinates is a rotation in $O(n)$. Thus the problem of determining whether two Riemnnian manifolds are isometric is reduced to finite dimensions. In Fefferman's setting the analogue of $O(n)$ is the group $\mathrm{H}^{+}$of all linear fractional transformations of $\mathbb{C}^{n}$ which preserve $\partial D_{0}$, where $D_{0}$ is an approximation of $D$. If $\Phi: D \rightarrow \tilde{D}$ is a biholomorphism, then the Moser normal form at $p \in \partial D$ corresponds to the Moser normal form at $\Phi(p)$ by an element of $H^{+}$. So, analogously to the Riemannian case, the problem of deciding whether two strictly convex domains are biholomorphic is reduced to finite dimensions. This leads to certain invariant polynomials related to the Moser normal form. The Bergman kernel on $D$ is analogous to a heatlike kernel, $K_{t}(x, y)$, on $M$. This $K_{t}$ has an asymptotic expansion, and $K_{t}(x, x)=$ $c_{n} t^{-\frac{n}{2}} \cdot\left\{1+\sum_{k+1} \gamma_{k}(x) t^{k}\right\}$, where the $\gamma_{k}$ are invariants determined by the Riemannian metric. Analogously for $D=\{\psi>0\}, K_{D}(z, z)=\phi(z) / \psi^{n+1}+\tilde{\phi}(z) \log \psi$. The Taylor expansions of $\tilde{\phi}$ and $\phi$ modulo $O\left(\psi^{n+1}\right)$ are uniquely determined by the Taylor expansion of $\partial D$. To carry out the analogy between the heat kernel and the Bergman kernel, Fefferman finds a function which is the analogue of $t$. This analogue is an approximate formal solution of the Monge-Ampère equation $J(u)=1$. To complete the analysis Fefferman must overcome enormous difficulties which arise from the fact that while Weyl's analysis is based on the semisimple group $O(n)$, Fefferman has to deal with the group $H^{+}$, which is not semisimple. This is not only an impressive technical feat but it introduces seminal original methods to the subject.

Among Fefferman's many important contributions to the study of strictly pseudoconvex domains is his 1985 joint paper with Harold Donnelly, "Fixed point formula for the Bergman kernel." They prove the following. Let $\Omega$ be a strictly pseudoconvex domain and $K(z, w)$ the associated Bergman kernel. Suppose that $\gamma: \Omega \rightarrow \Omega$ is a holomorphic automorphism having no fixed points on $\partial \Omega$. Then the fixed point set of $\gamma$ consists of a finite number of points $p_{1}, p_{2}, \ldots, p_{k}$. Denote by $\gamma_{* j}$ the holomorphic differential of $\gamma$ at the point $p_{j}$. Let $I$ denote the identity endomorphism and $J_{\gamma(z)}$ the holomorphic Jacobian of $\gamma$. Then

$$
\int_{\Omega} \overline{K(z, \gamma z)} J_{\gamma}(z) d z=\sum_{j} \frac{(-1)^{n}}{\operatorname{det}\left(I-\gamma_{* j}\right)} .
$$

This formula is then applied to study circle actions on $\Omega$.

Fefferman has made several contributions to geometry which are relevant to the understanding of CR structures, as in the 2003 joint paper with Kengo Hirachi, "Ambient metric construction of Q-curvature in conformal and CR geometries." All the work described above concerns 
the study of strictly pseudoconvex domains and CR manifolds. He has also made major contributions to the weakly convex case, which are described briefly below.

The local analysis of strictly pseudoconvex domains and CR manifolds is driven by the approximation of $D$ by $D_{0}$ mentioned above. In the general pseudoconvex case there is no analogous method. I have been privileged to collaborate with Fefferman in working on some of these problems. In a beautiful expository paper, "Kohn's microlocalization of $\bar{\partial}$ problems," Fefferman gives the background for our results. Our work deals with Hölder estimates for the operators $\bar{\partial}$ and $\bar{\partial}_{b}$, the associated Laplacians, and projection operators. The starting point for the analysis of $\bar{\partial}_{b}$ on $\partial D$ and on CR manifolds is the local subelliptic estimate

$$
\|\varphi\|_{\varepsilon}^{2} \leq C\left(\left\|\bar{\partial}_{b} \varphi\right\|^{2}+\left\|\bar{\partial}_{b}^{*} \varphi\right\|^{2}\right),
$$

where the $\varphi$ are $(0,1)$-forms in $C_{0}^{\infty}(U)$ in an open set $U$. For the analysis of $\bar{\partial}$ the starting point is an analogous estimate on $C_{0}^{\infty}(U \cap \bar{D})$ with appropriate boundary conditions on $U \cap \partial D$. These estimates imply $C^{\infty}$ regularity. The problem is to prove Hölder regularity. This regularity is proved in the special case where the Levi form is locally diagonalizable, which means that the Levi form can be expressed in terms of local tangential $(1,0)$ vector fields $L_{1}, \ldots, L_{n-1}$ by $c_{i j}=\mathcal{L}\left(L_{i}, \bar{L}_{j}\right)$, and it is diagonalizable if there exist $L$ s so that $c_{i j}=\lambda_{i} \delta_{i j}$. Diagonalizability clearly holds when $n=2$, but in case $n>2$ it is a severe restriction. This work and an extension to the threedimensional case is contained in a collection of joint papers (one including M. Machedon) published in 19881990. In the general nondiagonalizable case it remains a difficult problem to prove Hölder estimates. The first obstacle is that in these cases the subelliptic estimates do not fit the CR structure, and thus different and much stronger estimates are needed for a starting point. In Fefferman's remarkable paper "The uncertainty principle" and in subsequent work with D. H. Phong, powerful new methods of proving a priori estimates in PDE are established. These give insights into the type of problems discussed here and, hopefully, will lead to a solution.

\section{Sun-Yung Alice Chang and C. Robin Graham}

\section{Fefferman's Work in Conformal Geometry}

As Charlie's colleagues and collaborators, we feel privileged to have had the opportunity to work with him. His contributions to conformal geometry are centered around the ambient metric construction. We begin with a discussion of some background leading up to its discovery.

The conformal ambient metric grew out of Charlie's work in several complex variables, in particular in his

Sun-Yung Alice Chang is professor of mathematics at Princeton University. Her email address is chang@math. princeton.edu. C. Robin Graham is professor of mathematics at the University of Washington. His email address is robin@math . washington. edu.

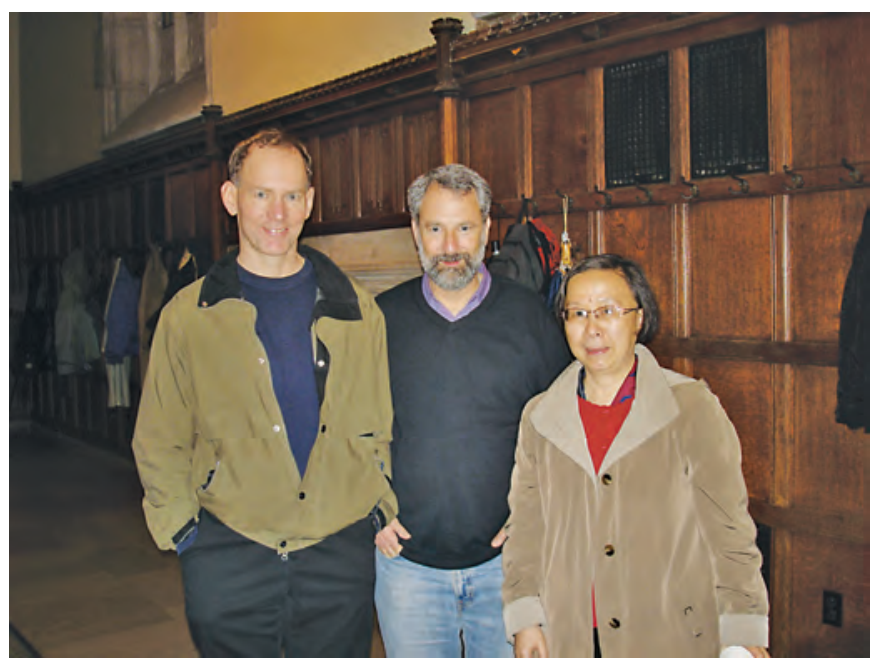

C. Robin Graham, Charlie Fefferman, and Sun-Yung Alice Chang in 2009 at a conference in Charlie's honor.

efforts to understand the asymptotic expansion of the Bergman kernel. As Charlie had previously shown, the restriction to the diagonal of the Bergman kernel $K$ of a smooth, bounded strictly pseudoconvex domain $\Omega \subset \mathbb{C}^{n}$ can be written in the form

$$
K(z, z)=\varphi(z) \rho(z)^{-n-1}+\psi(z) \log \rho(z),
$$

where $\rho$ is a smooth defining function for $\partial \Omega$ and $\varphi$, $\psi \in C^{\infty}(\bar{\Omega})$. This expansion can be viewed by analogy to the asymptotic expansion of the heat kernel of a Riemannian manifold restricted to the diagonal. The heat kernel can be expanded in powers of the time variable $t$, and the coefficients in the expansion are local scalar invariants of Riemannian metrics, which can be constructed as contractions of tensor products of covariant derivatives of the curvature tensor. The Bergman kernel on the diagonal is determined locally by the boundary up to a smooth function, so it was natural to try to find an analogous expansion for $\varphi$ to order $n+1$ and for $\psi$ to infinite order. But several problems immediately arose in contemplating carrying this out. One was that $\Omega$ is not canonically a product near $\partial \Omega$, so there was no obvious analogue of $t$, nor was there an obvious way to formulate an expansion in such a way that the coefficients would be geometric invariants of the boundary. But even if one could surmount these difficulties, the most glaring problem was the fact that it was not known how to construct general scalar invariants of CR structures, the geometric structures induced on nondegenerate hypersurfaces by the complex structure on the background $\mathbb{C}^{n}$.

Charlie resolved these difficulties in his groundbreaking paper "Parabolic invariant theory in complex analysis." His solution was to construct a Lorentz signature, asymptotically Kähler-Einstein metric $\tilde{g}$ on $\mathbb{C}^{*} \times \Omega$, where $\mathbb{C}^{*}=\mathbb{C} \backslash\{0\}$, via a formal solution of a degenerate complex Monge-Ampère equation. This metric $\tilde{g}$ is invariant under 
rotations and homogeneous under dilations in $\mathbb{C}^{*}$ and is invariantly associated to the $\mathrm{CR}$ geometry on $\partial \Omega$. The formal solution to the Monge-Ampère equation plays the role of the time variable $t$, and scalar CR invariants can be constructed using the curvature tensor and the LeviCivita connection of $\tilde{g}$ in a manner roughly analogous to the case of Riemannian geometry. There are more details of this solution in Joseph Kohn's section.

The conformal ambient metric, introduced in joint work with C. R. Graham, "Conformal invariants," and also denoted $\tilde{g}$, is an analogue in a different setting: it is determined by the datum of a conformal class $(M,[g])$ of Riemannian metrics on a manifold $M$ of dimension $n \geq 3$. Metrics in the conformal class are sections of a ray subbundle $G$ of the bundle of symmetric 2-tensors on $M$, and $\tilde{g}$ is a Lorentz signature metric on the

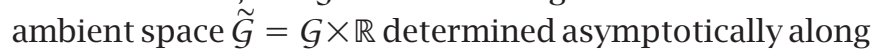
$G \cong G \times\{0\}$. The model is the sphere $S^{n}$, whose group of conformal motions is the Lorentz group $O(n+1,1)$ of linear transformations of $\mathbb{R}^{n+2}$ preserving a quadratic form of signature $(n+1,1)$. The ray bundle $\mathcal{G}$ can be identified with the forward pointing half of the null cone of the quadratic form. The ambient metric for the sphere is just the Minkowski metric on $\mathbb{R}^{n+2}$, which is clearly preserved by the conformal motions in $O(n+1,1)$ in their linear action on $\mathbb{R}^{n+2}$. For a general conformal class of metrics $(M,[g])$, the ambient metric is a Lorentz signature metric on $\widetilde{\mathcal{G}}$ determined asymptotically along $\mathcal{G} \times\{0\}$ by the following three conditions:

(i) $\tilde{g}$ is homogeneous of degree two with respect to natural dilations on $\widetilde{\mathcal{G}}$,

(ii) $\iota^{*} \tilde{g}=\mathbf{g}_{0}$,

(iii) $\operatorname{Ric}(\tilde{g})$ vanishes asymptotically at $G \times\{0\}$.

Here $\mathbf{g}_{\mathbf{0}}$ is a tautological symmetric 2-tensor on $G$ determined by the conformal class, and $\iota: G \rightarrow G \times\{0\} \subset G \times \mathbb{R}$ is the inclusion. In (iii) the asymptotic order of vanishing is infinite if $n$ is odd and is $n / 2-1$ if $n$ is even. The ambient metric $\tilde{g}$ is uniquely determined up to diffeomorphism by these conditions: to infinite order if $n$ is odd and to order $n / 2$ if $n$ is even.

The conformal ambient metric was inspired by Charlie's construction in several complex variables, but the relationship is closer than mere analogy: the Kähler-Lorentz metric in the several complex variables construction can be viewed as a special case of a conformal ambient metric. One takes the conformal class to be the so-called "Fefferman metric" (there are too many of these!) on $\partial \Omega \times S^{1}$, which Charlie had constructed earlier. Although one usually thinks of conformal geometry as simpler than CR geometry, by this construction the class of CR structures induced on nondegenerate boundaries of domains in $\mathbb{C}^{n}$ can be viewed as a subclass of the class of conformal structures on even-dimensional manifolds.

There is a second, equivalent formulation of ambient metrics, namely, as Poincaré metrics. The model here is hyperbolic space. Recall that $S^{n}$ can be viewed as the boundary at infinity of hyperbolic space, which arises as the restriction of the Minkowski metric on $\mathbb{R}^{n+2}$ to one sheet of the hyperboloid arising as the -1-level set of the Lorentz signature quadratic form. This construction generalizes to the case of a general conformal manifold as the "conformal infinity"; the ambient metric can be restricted to a natural hypersurface in $\widetilde{\mathcal{G}}$, and the resulting Poincaré metric has asymptotically constant negative Ricci curvature.

The
ambient
metric
opened up
new arenas
for study in
geometric
analysis.

These constructions have been enormously influential; the ambient and Poincaré metrics are now viewed as fundamental in conformal geometry and beyond. As described above, one of Charlie's original motivations for the construction in CR geometry was to construct and characterize scalar invariants of CR structures to describe the asymptotic expansion of the Bergman kernel. The ambient metric enables construction of scalar conformal invariants as Weyl invariants, constructed as linear combinations of complete contractions of covariant derivatives of the curvature tensor of the ambient metric. Determining the extent to which all invariants arise by this construction involved developing a new parabolic invariant theory; this was carried out in joint work with Graham and by Graham with Bailey and Eastwood.

The ambient metric opened up new arenas for study in geometric analysis. Conformally invariant powers of the Laplacian were constructed in terms of the ambient metric, leading to Branson's construction of $Q$-curvature. $Q$-curvature is a higher-dimensional version of scalar curvature in dimension 2. Branson's original definition proceeded by analytic continuation in the dimension, and $Q$ curvature was originally regarded as rather mysterious. Charlie, in separate papers with Graham and Hirachi in the first years of this century, helped illuminate its nature. $Q$ curvature enters into the Gauss-Bonnet integrand in higher dimensions (albeit in a more complicated way than the scalar curvature in dimension 2). In dimension 4 and modulo the part which is pointwise conformally invariant, the Gauss-Bonnet integrand has fully nonlinear structure under conformal change of metric. The analytic study of such partial differential equations has become a central topic in conformal geometry.

The fundamental idea underlying the ambient/Poincaré metric is to study geometry in dimension $n$ by passing to a different but essentially equivalent description in dimension $n+1$ or $n+2$. The AdS/CFT correspondence in physics, a major development since its introduction by Maldacena in 1997, is based on the same idea. In fact, the Poincaré metric construction amounts to the geometry underlying the AdS/CFT duality between conformal field theories on a boundary at infinity and supergravity in the bulk. This synergy between geometry and physics has stimulated both fields and continues to be a source of exciting developments today. 


\section{Diego Córdoba}

\section{Fefferman on Fluid Dynamics}

The search for singularities in incompressible fluids has become a major challenge in the area of nonlinear and nonlocal partial differential equations. In particular the existence, or absence, of finite-time singularities with finite energy remains an open problem for 3D incompressible Euler equations in the whole domain $\mathbb{R}^{3}$ or in the periodic $\mathbb{T}^{3}$ setting. The local existence in time of classical solutions is well known, and in dimension two these classical solutions exist for all time. A result of Beale, Kato, and Majda asserts that if a singularity forms at time $T$, then the vorticity $\omega(x, t)$ grows so rapidly that

$$
\int_{0}^{T} \sup _{x}|\omega(x, t)| d t=\infty
$$

Notice that in dimension two the vorticity $\omega$ remains bounded, since it is transported by the flow; this is a main difference with the situation in dimension three.

Fefferman's interest in fluid dynamics started at the beginning of the 1990s in a collaboration with Constantin and Majda. They showed that if the velocity remains bounded up to the time of singularity formation, then the vorticity direction $\frac{\omega(x, t)}{|\omega(x, t)|}$ cannot remain uniformly Lipschitz continuous up to that time. A similar result was proven in the presence of viscosity. This result is extremely useful, since vortex lines are transported by the 3D incompressible Euler flow. Recall that a vortex line in a fluid is an arc on an integral curve of the vorticity $\omega(x, t)$ for fixed $t$. In numerical simulations of 3D Euler solutions, one routinely sees that vortex tubes (tubular neighborhoods arising as a union of vortex lines) grow longer and thinner while bending and twisting. In particular, if the thickness of a piece of a vortex tube becomes zero in finite time, then one has a singular solution of 3D Euler. I collaborated with Charlie to prove that given a criterion only at the level of the velocity field then a vortex tube cannot reach zero thickness in finite time, unless it bends and twists so violently that no part of it forms a "smooth" tube anymore. If additionally we add the assumption that there is a uniform collapse, in the sense that the maximum and the minimum thickness are comparable, then we can obtain a lower bound on the rate of decay of the thickness of the tube.

A key ingredient in the success of proving the formation of singularities is to identify a scenario in which there is a clear mechanism developing fast vorticity growth, and accurate numerics plays a crucial role in this search. Below we describe two plausible singular scenarios discovered first by numerical simulations which led later to a rigorous proof: splash singularities for water waves and shift stability for the dynamics of the interface between two immiscible incompressible fluids in a porous medium.

Diego Córdoba is professor of mathematics at the Spanish National Research Council (CSIC-ICMAT). His email address is dcg@icmat.es.

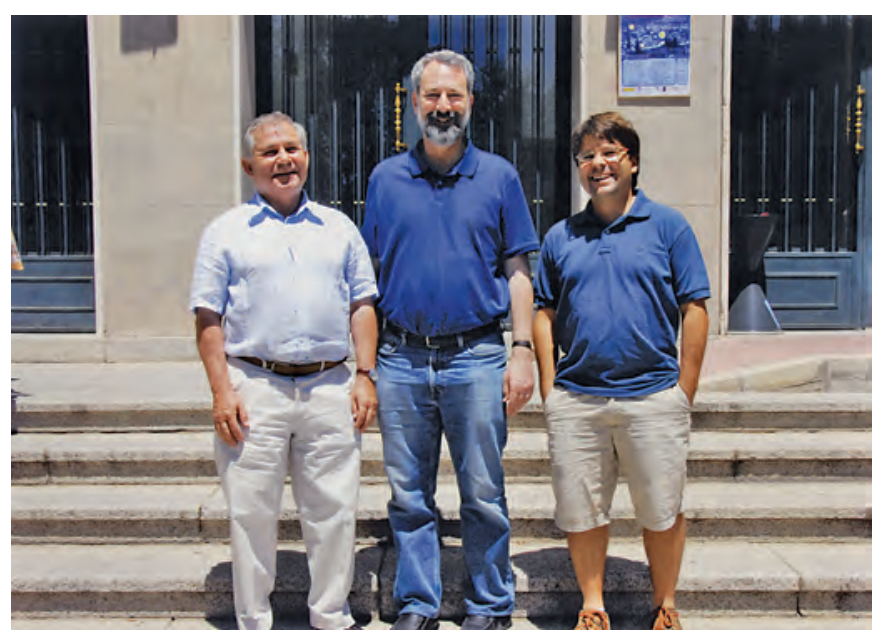

Charlie with Antonio Córdoba and Antonio's son, Diego Córdoba, both PhD students of Fefferman.

\section{Splash and Splat Singularities}

In the case of the incompressible Euler equations with a free boundary, Charlie, in collaboration with Castro, Gancedo, Gómez-Serrano, and me, established the formation in finite time of splash and splat singularities for the water wave problem. A splash singularity appears when the free boundary remains smooth but self-intersects at a point; a splat singularity is when it self-intersects along an arc. The difference with the fixed domain case is that the pressure is constant at the boundary, and the boundary moves with the velocity of the fluid. Therefore finding the domain of the fluid is part of the problem. The main idea of the proof is to choose a conformal map $\phi$ which separates the point of collapse, such that its singular points (where $\phi$ cannot be inverted) are located outside the domain of the fluid. This map transforms the splash into a closed curve whose chord-arc is well defined. We can select an initial velocity that immediately separates the point of collapse because the equations are reversible in time and we can solve backwards in time. In order to return to the original domain and obtain solutions to the free boundary incompressible Euler equations, it is necessary to invert the map $\phi$. In the presence of viscosity the proof has to be modified since the equations are no longer reversible; we use again the conformal map $\phi$ that separates the self-intersecting points of the splash curve. But instead of showing local existence backwards in time in the transformed domain, we prove local existence forward in time and show that the solutions depend stably on the initial conditions. We apply a perturbative argument to prove a splash, but not a splat, singularity for Navier-Stokes.

In recent work Fefferman, in collaboration with Ionescu and Lie, showed that the presence of a second fluid prevents the formation in finite time of both splash and splat singularities. The condition that the densities of the fluids are positive is used to show a critical $L^{\infty}$ bound for the measure of the vorticity in the boundary which 
prevents self-intersections of the boundary between the fluids.

\section{Shift of Stability and Breakdown}

Charlie has also worked on the dynamics of the interface between two incompressible viscous fluids with different densities in a porous medium, which is modeled by Darcy's law. This problem is also known as the Muskat problem. If the heavier fluid is below the light fluid the system is well posed in a Sobolev space $H^{k}$, but if the interface is not a graph the system is unstable. Together with collaborators Castro, Gancedo, and López-Fernández we have proven that there exists a nonempty open set of initial data in the stable regime (heavier fluid below the light fluid for which the interface is initially in $H^{4}$ ), such that the solution of the Muskat problem becomes immediately real-analytic and then passes to the unstable regime in finite time. Moreover, the Cauchy-Kowalewski theorem shows that a real-analytic Muskat solution continues to exist for a short time after the turnover. The interface becomes more and more unstable as the turnover progresses. In fact, there exist interfaces of the Muskat problem such that after turnover their smoothness breaks down. The proof follows by a rigorous analysis of the full nonlinear problem and establishing analytic continuation of Muskat solutions to the time-varying strip of analyticity.

\section{Bo'az Klartag}

\section{Fefferman's Work on the Whitney Extension Problem}

Given an arbitrary set $E \subseteq \mathbb{R}^{n}$ and a function $f: E \rightarrow \mathbb{R}$ and an $m \geq 1$, does there exist a $C^{m}$-smooth function $F: \mathbb{R}^{n} \rightarrow \mathbb{R}$ that extends $f$ (i.e., $\left.F\right|_{E}=f$ )? A problem attributed to Hassler Whitney seeks to find plausible necessary and sufficient conditions for the feasibility of such a $C^{m}$-extension.

In the 1930s Whitney proved that the function $f$ extends to a $C^{m}$-smooth function on the entire real line if and only if for any accumulation point $x$ of the set $E$, the difference quotient of order $m$,

$$
\left[x_{0}, \ldots, x_{m}\right](f):=m ! \cdot \sum_{i=0}^{m}\left(\prod_{j \neq i} \frac{1}{x_{i}-x_{j}}\right) f\left(x_{i}\right),
$$

converges to a finite limit as the distinct points $x_{0}, \ldots, x_{m} \in$ $E$ tend to the point $x$. This finite limit will be the $m^{\text {th }}$ derivative at $x$ of any $C^{m}$-smooth function that extends $f$. In fact, $\left[x_{0}, \ldots, x_{m}\right](f)$ equals the $m^{\text {th }}$-derivative of the Lagrange interpolation polynomial $P$ of degree $m$ with $P\left(x_{i}\right)=f\left(x_{i}\right)$ for all $i$.

The extension problem in $\mathbb{R}^{n}$ for $n \geq 2$ is more difficult, as there could be many potential candidates for the $m^{\text {th }}$-order Taylor polynomial of the extension function.

Bo'az Klartag is professor of mathematics at Tel Aviv University. His email address is k1artag@tav.ac. 11.

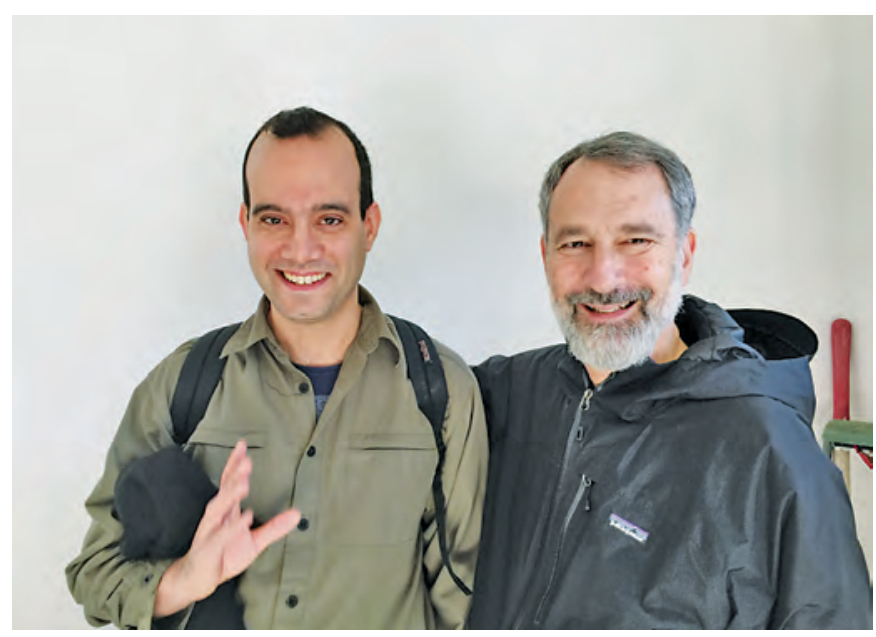

Bo’az Klartag and Charlie at Princeton circa 2015.

We first discuss the case of $C^{m, 1}$-functions, which are $C^{m}$-smooth functions $F: \mathbb{R}^{n} \rightarrow \mathbb{R}$ for which the norm

$$
\begin{aligned}
\|F\|_{C^{m, 1}}:= & \sup _{x \in \mathbb{R}^{n}|\alpha| \leq m} \max _{\left|\partial^{\alpha} F(x)\right|} \mid \partial^{\alpha} \\
& +\sup _{x \neq y \in \mathbb{R}^{n}|\alpha|=m} \max _{x \neq} \frac{\left|\partial^{\alpha} F(x)-\partial^{\alpha} F(y)\right|}{|x-y|}
\end{aligned}
$$

is finite. Here, the multiindex $\alpha=\left(\alpha_{1}, \ldots, \alpha_{n}\right)$ is a vector of nonnegative integers, $\partial^{\alpha} F(x)=\left(\frac{\partial}{\partial x_{1}}\right)^{\alpha_{1}} \cdots\left(\frac{\partial}{\partial x_{n}}\right)^{\alpha_{n}} F(x)$ and $|\alpha|=\sum_{i} \alpha_{i}$. In a seminal work from the early 2000s, Charlie Fefferman proved the following finiteness principle, which had been conjectured earlier by Brudnyi and Shvartsman:

Theorem 1. Let $n \geq 1, m \geq 0$, and let $E \subseteq \mathbb{R}^{n}$ and $f: E \rightarrow$ $\mathbb{R}$ be arbitrary. Assume that there exists $M>0$ with the following property: For any finite subset $S \subseteq E$ of size at $\operatorname{most} k(m, n)<\infty$, the function $\left.f\right|_{S}$ extends to an auxiliary $C^{m, 1}$-function $F_{S}: \mathbb{R}^{n} \rightarrow \mathbb{R}$ with $\left\|F_{S}\right\|_{C^{m, 1}} \leq M$. Then there exists a $C^{m, 1}$-function $F: \mathbb{R}^{n} \rightarrow \mathbb{R}$ with $\left.F\right|_{E}=f$. Moreover, $\|F\|_{C^{m, 1}} \leq C M$ for some constant $C=C(m, n)$.

Thus in order to tell whether $f$ extends to a $C^{m, 1}$. function on the entire $\mathbb{R}^{n}$, it suffices to consider subsets of $E$ with at most $k(m, n)$ elements. The optimal finiteness constant $k(m, n)$ is not known in general.

Merely a year or two following his proof of Theorem 1 , Fefferman was able to resolve Whitney's $C^{m}$-extension problem in another remarkable work. The reader is referred to Fefferman's 2009 Bulletin article on the topic for a precise formulation of his $C^{m}$-theorem, which involves the notion of Glaeser refinement of certain fiber bundles over an arbitrary set $E \subseteq \mathbb{R}^{n}$, where the fiber of the bundle at a base point $x \in E$ consists of potential Taylor polynomials at $x$ of a $C^{m}$-extension function. This form of Fefferman's solution is closely related to an earlier work by Bierstone, Milman, and Pawłucki dealing with a subanalytic set $E$. Glaeser himself settled the case $m=1$ in the 1950s.

The next question is whether one can turn Fefferman's constructive proof into an actual algorithm for a nearly 
optimal $C^{m}$-interpolation of data. From the viewpoint of computer science, the problem may be formulated as follows. We are given a large, finite subset $E \subset \mathbb{R}^{n}$ and a function $f: E \rightarrow \mathbb{R}$. For $m \geq 1$ define

$$
\begin{aligned}
& \|f\|_{C^{m}(E)}=\inf \left\{\|F\|_{C^{m}\left(\mathbb{R}^{n}\right)} ;\right. \\
& \left.\qquad F: \mathbb{R}^{n} \rightarrow \mathbb{R} \text { is } C^{m} \text {-smooth with }\left.F\right|_{E}=f\right\},
\end{aligned}
$$

where $\|F\|_{C^{m}\left(\mathbb{R}^{n}\right)}=\|F\|_{C^{m-1,1}}$ for a $C^{m}$-smooth function $F: \mathbb{R}^{n} \rightarrow \mathbb{R}$. Our goal is to compute, using an (idealized) digital computer, a function $F: \mathbb{R}^{n} \rightarrow \mathbb{R}$ with $\left.F\right|_{E}=f$ and $\|F\|_{C^{m}\left(\mathbb{R}^{n}\right)} \leq C\|f\|_{C^{m}(E)}$, for $C>0$ being a constant depending only on $m$ and $n$. In a joint work with Klartag, Fefferman has transformed his proof of Theorem 1 into an algorithm with the following interface:

(i) First, the user enters the entire data set (the coordinates of the points of $E$ and the values of $f$ ) into the computer. The computer then works for a while, performing at most $C N \log N$ operations where $N=\#(E)$, after which it signals that it is ready to accept further input.

(ii) Then, whenever the user enters the coordinates of a point $x \in \mathbb{R}^{n}$, the computer rapidly responds, using only $C \log N$ operations with the value $F(x)$.

The function $F$ that is computed by the algorithm is guaranteed to be an extension of $f$, with $\|F\|_{C^{m}\left(\mathbb{R}^{n}\right)} \leq$ $C\|f\|_{C^{m}(E)}$. The storage required by the algorithm is bounded by $C N$. Of course, the handling of real numbers in the digital computer is only up to finite precision; the algorithm returns its answers in the given precision of the digital computer. The reader might wonder why $C N \log N$ operations suffice when Theorem 1 requires an inspection of all subsets of $E$ of size $k(m, n)$. As it turns out, there exists a list of no more than $C N$ subsets, each of size no greater than $C$, that matter, as proven by Fefferman. Extensions of his result exist for smooth selection and for interpolating multidimensional functions with noisy data. Particularly notable is the extension to Sobolev spaces; in a series of papers Fefferman, Israel, and Luli proved the existence of an extension and described an algorithm to construct it. That algorithm shares features with the $C^{m}$-algorithm described above: the preprocessing time is $C N \log N$, and the query time is only $C \log N$.

We move on to discuss some of Fefferman's work on the $(1+\varepsilon)$-version of the quantitative Whitney problem, where one is given a small $\varepsilon>0$ and is seeking a $C^{m}$-function $F: \mathbb{R}^{n} \rightarrow \mathbb{R}$ extending the given function $f: E \rightarrow \mathbb{R}$ such that

$$
\|F\|_{C^{m}\left(\mathbb{R}^{n}\right)} \leq(1+\varepsilon) \cdot\|f\|_{C^{m}(E)} .
$$

The $C^{m}\left(\mathbb{R}^{n}\right)$-norm is usually considered only up to a multiplicative constant; some authors define the $C^{m}$ and $C^{m, 1}$-norms a bit differently, replacing the sum in (8) by a maximum. Nevertheless, from the point of view of data interpolation, it makes sense to fix a reasonable $C^{m}$-norm and ask for an efficient algorithm that produces a function $F$ satisfying (9), given a finite set $E \subseteq \mathbb{R}^{n}$ and function values $f: E \rightarrow \mathbb{R}$.

Fefferman devised a polynomial-time algorithm for carrying out this task for all $m$ and $n$. In the case of
$C^{2}\left(\mathbb{R}^{2}\right)$, the preprocessing time is at most $C(\varepsilon) N \log N$, the query work is $C \log (N / \varepsilon)$, and the storage $C(\varepsilon) N$. Moreover, Fefferman has also come up with a $(1+\varepsilon)$ version of Whitney's extension theorem, whose proof uses the notion of a "gentle partition of unity." For simplicity, we restrict our attention to the central case of a finite set $E$ in the following formulation of his theorem:

Theorem 2. Let $m, n \geq 1$ and $\varepsilon>0$ be given, and let $E \subseteq$ $\mathbb{R}^{n}$ be a finite set. Let $\left(P_{x}\right)_{x \in E}$ be a family of polynomials of degree $m$ in $n$ real variables.

Assume that for any $S \subseteq E$ with $\#(S) \leq k^{\#}(m, n, \varepsilon)$ there exists an auxiliary $C^{m}$-smooth function $F_{S}: \mathbb{R}^{n} \rightarrow \mathbb{R}$ with $\left\|F_{S}\right\|_{C^{m}\left(\mathbb{R}^{n}\right)} \leq 1$, such that for any $x \in S$, the $m^{\text {th }}$-order Taylor polynomial of $F_{S}$ at the point $x$, denoted by $J_{X}\left(F_{S}\right)$, satisfies $J_{X}\left(F_{S}\right)=P_{X}$. Then there exists a $C^{m}$-smooth function $F: \mathbb{R}^{n} \rightarrow \mathbb{R}$ with $J_{x}(F)=P_{x}$ for all $x \in E$ such that $\|F\|_{C^{m}\left(\mathbb{R}^{n}\right)} \leq 1+\varepsilon$.

We could go on and on. Only space limitations prevent us from describing additional Whitney-related theorems that were published by Fefferman in recent years. We would like to conclude by wishing Charlie and the mathematical community many more beautiful results on the subject in years to come!

Postscript: In June 2017 Fefferman and Shvartsman announced their proof of a finiteness principle for Lipschitz selection in an arbitrary metric space. That is, assume that we are given a metric space $X$ and a compact, convex set $K_{X} \subseteq \mathbb{R}^{d}$ associated with any point $x \in X$. Assume that for any subset $S \subseteq X$ with $\#(S) \leq 2^{d}$ there exists a 1-Lipschitz map $F_{S}: S \rightarrow \mathbb{R}^{d}$ with $F_{S}(x) \in K(x)$ for all $x \in S$. Then there exists a $C$-Lipschitz map $F: X \rightarrow \mathbb{R}^{d}$ with $F(x) \in K(x)$ for all $x \in X$, where $C$ is a constant depending only on $d$.

\section{Jürg Fröhlich, Luis Seco, and Michael Weinstein}

\section{Charlie's Romance with Quantum Theory}

Our memories of Charlie Fefferman go back to the middle of the seventies when Jürg and Charlie arrived at Princeton. Barry Simon, then at Princeton, had announced that he had a "secret weapon" that might well enable him to construct models of local relativistic quantum fields in four-dimensional Minkowski space-a dream of many mathematical physicists working in constructive quantum field theory, yet to come true. It turned out that Barry's secret weapon was Charlie! He was convinced that if Charlie were willing to put his mind to problems in relativistic quantum field theory success in solving

Jürg Fröhlich is professor in the Institute for Theoretical Physics at The Swiss Federal Institute of Technology (ETH) Zürich. His email address is juerg@phys. ethz.ch.

Luis Seco is professor of mathematics at the University of Toronto. His email address is seco@math. utoronto.ca.

Michael Weinstein is professor of mathematics and professor of applied mathematics at Columbia University. His email address is miw2103@columbia.edu. 
them would soon be within reach. Alas, Barry's hopes did not materialize. Luckily we all recovered from this mishap and went on to solve other interesting, albeit somewhat less challenging, problems in quantum theory. For example, Charlie and his former student Antonio Córdoba developed the so-called wave-packet transform, an analytical tool built upon Heisenberg's uncertainty relations.

There followed a long period when Jürg's and Charlie's trajectories did not intersect. In the mid-eighties, after Jürg had moved back to ETH in Zürich, he heard of an exciting discovery: Charlie had solved the problem of understanding why, at appropriate temperatures and densities, atomic nuclei and electrons form gases of

...his
distinctive
style in
analysis: at
once
powerful,
bare-hands,
and elegant
neutral atoms or molecules. His result appeared under the title "The atomic and molecular nature of matter" in the first issue of the first volume of a new journal that A. Córdoba had founded, Rev. Mat. Iberoamericana. The abstract of the paper reads: "The purpose of this article is to show that electrons and protons, interacting by Coulomb forces and governed by quantum statistical mechanics at suitable temperature and density, form a gas of Hydrogen atoms or molecules."

At around that time, Luis arrived at Princeton as a graduate student. He was closely familiar with Charlie's previous work on harmonic analysis, but he had no idea what an electron was. One of the unwritten rules of the Princeton mathematics department is that it does not offer graduate courses on subjects where there is already a good book. As a consequence, professors teach courses on whatever topic they just spent their time working on, and it is not unusual that some of their colleagues attend the lectures.

In September 1984 Michael introduced himself to Charlie as they were riding on a Fine Hall elevator and said he was new at Princeton. Charlie introduced himself to Michael and said he had been there forever. He gave Michael his coordinates and encouraged him to stop by to chat. That semester Michael followed Charlie's course, advertised in the catalogue as Fourier Analysis on Groups, which turned out to be about Charlie's ongoing work on stability of matter, treated quantum-mechanically, first established by Dyson and Lenard and later by Lieb and Thirring and others. His course was also attended by another assistant professor, Rafael de la Llave, who was Charlie's collaborator in his work "Relativistic stability of matter." It was through these wonderful lectures that Charlie introduced Michael to deep mathematical problems in quantum theory and to his distinctive style in analysis: at once powerful, bare-hands, and elegant. And then there was Luis, taking his first course with Charlie after arriving at Princeton.
Here is what stability of matter means: Consider a system in physical space $\mathbb{R}^{3}$ consisting of $M$ static nuclei at positions $y_{j}$ with atomic numbers (charges) $Z_{j}$, $j=1, \ldots, M$, and $N$ electrons at positions $x_{k}, k=1, \ldots, N$, interacting through Coulomb forces. The Hamiltonian operator of such a system is given by

$$
H_{Z, M, N}=\sum_{k=1}^{N}\left(-\Delta_{X_{k}}\right)+V_{Z, M, N}
$$

The Laplacian terms represent the kinetic energy of the electrons (their mass being set to $1 / 2$ ), while the second term on the right side is the Coulomb potential energy, which is given by the multiplication operator

$$
\text { (11) } V_{Z, M, N}=\sum_{j<k} \frac{1}{\left|x_{j}-x_{k}\right|}+\sum_{j<k} \frac{Z_{j} Z_{k}}{\left|y_{j}-y_{k}\right|}-\sum_{j, k} \frac{Z_{k}}{\left|x_{j}-y_{k}\right|} \text {. }
$$

The Hamiltonian $H_{Z, M, N}$ acts on the Hilbert space $\mathcal{H}_{N}$ given by the $N$-fold antisymmetric tensor product of the one-electron Hilbert space, $L^{2}\left(\mathbb{R}^{3}, \mathrm{~d}^{3} x\right) \otimes \mathbb{C}^{2}$, where $L^{2}\left(\mathbb{R}^{3}, \mathrm{~d}^{3} x\right)$ is the space of orbital wave functions of an electron and $\mathbb{C}^{2}$ is the space of its spin states.

One also considers the pseudo-relativistic version of the Hamiltonian, with $H_{Z, M, N}$ now given by

$$
H_{Z, M, N}=\sum_{k=1}^{N}\left(-\Delta_{\chi_{k}}\right)^{1 / 2}+\alpha \cdot V_{Z, M, N} .
$$

In this operator the fine structure constant $\alpha \simeq 1 / 137$ appears as a fundamental parameter, and (12) comes with other mathematical licenses that turn the original physical problem into this form, keeping the essential feature that, in relativity theory, kinetic energy is proportional to momentum in the high-energy limit.

Stability of matter is the property that

$$
\left\langle H_{Z, M, N} \psi, \psi\right\rangle \geq-C_{Z} \cdot(M+N)
$$

in the nonrelativistic case (10), and

$$
\left\langle H_{Z, M, N} \psi, \psi\right\rangle \geq 0
$$

for the relativistic Hamiltonian (12), where $\psi$ is an arbitrary wave function in $\mathcal{H}_{N}$ of norm 1 . In the latter case, the critical role of the fine structure constant becomes clear after one realizes that in (12), the Coulomb potential energy and the relativistic kinetic energy scale in the same way, namely, as inverse lengths. Thus, either (14) holds or $\inf \left\langle H_{Z, M, N} \psi, \psi\right\rangle=-\infty$. If the bounds (13), (14), respectively, did not hold true one would conclude that matter must collapse and, in the process, release a devastating amount of energy.

Charlie's first landmark achievement in quantum theory was his work with Rafael de la Llave on stability of matter for Coulomb systems described by (10) and their pseudorelativistic cousins, as described by (12). Needless to say, Luis understood nothing of all this, except for one thing: in the relativistic case, Fefferman's philosophy was that, since the scaling properties of the operator in (12) make the problem hard, one had better exploit these properties to one's advantage by rewriting the kinetic 
energy and the Coulomb potential in a convenient form, as follows:

(15)

$\frac{1}{|x|}=\frac{1}{\pi} \iint_{R>0, z \in R^{3}}\left\{\begin{array}{ll}1 & \text { if both } x, 0 \in B(z, R), \\ 0 & \text { otherwise }\end{array}\right\} \frac{d z d R}{R^{5}}$,

with a similar expression for the Laplacian term. This expression is, in some sense, obvious, since the left and right sides scale in the same way (as an inverse length) and both are translation invariant. The revolutionary insight here is that the left-hand side is hard, but the right-hand side is easy: all it requires is counting the number of electrons or nuclei in a ball. Equation (15) turned out to be a game-changing element, with the strong flavor of microlocal analysis. This attracted Luis to make a move that would seem absurd to an outsider: to do a thesis on mathematical physics. Luis's move began a fruitful collaboration that would last a decade, involved very smart discussions, on-going oversight by de la Llave, and produced a long list of results on Coulomb systems. Their work concerned single atoms - to be precise, atoms with large atomic number $Z$, setting $M=1$ in (10). The ground-state energy of such atoms is given by

$$
E(Z, N)=\min _{\psi \in \mathcal{H}_{N},\|\psi\|=1}\left\langle H_{Z, N} \psi, \psi\right\rangle .
$$

They adopted the asymptotic perspective that $Z \rightarrow \infty$. While, in nature, $Z$ does not get to be much bigger than 100 , the Hamiltonian acts on functions that can easily depend on several hundred variables. Much of the mathematical complexity therefore comes from dimensionality considerations that will recreate the flavor of the semiclassical limit, WKB theory, spectral asymptotics, and the lattice-point problem of number theory.

Their first achievement was a proof of asymptotic neutrality of large ions, proving that the number $N(Z)$ of electrons that renders $E(Z, N)$ minimal,

$$
E(Z)=E(Z, N(Z))=\min _{N} E(Z, N),
$$

satisfies the asymptotic expression

$$
N(Z)=Z+O\left(Z^{0.84}\right) .
$$

The key ingredient was to perform a deep, rigorous benchmarking of the atomic Hamiltonian with its semiclassical version, the well-known Thomas-Fermi theory. This strategy was repeated in several different papers-in one notable example to obtain asymptotic estimates for the total spin of an atom-but its climax was reached in 1991 when Fefferman and Seco announced their proof of the Dirac-Schwinger conjecture, a long-standing open problem on the altar of mathematical physics:

$$
E(Z)=-c_{T F} Z^{7 / 3}+c_{S} Z^{2}+c_{D S} Z^{5 / 3}+o\left(Z^{5 / 3}\right) .
$$

Each term above is loaded with history, and the last term also signified its last chapter. Its derivation is deep and long, involving interesting elements of number theory and computer assistance. That number theory becomes relevant in the semiclassical limits is easy to understand: the lattice-point problem is encountered in the spectral asymptotics of the Laplacian. Without this fact, the term proportional to $Z^{5 / 3}$ in (16) would be multiplied by an oscillatory term depending on $Z$. This paints a number-theoretical perspective on the periodic table of elements.

As these papers on atomic physics were being finished, in the first half of the nineties, Charlie announced that he would visit ETH. He told Jürg that he would like to meet him and find out what he was up to. Jürg told Charlie that he had a PhD student ${ }^{1}$ whom he had encouraged to work on a problem that had interested him for well over a decade:

Consider the quantum-mechanical description of a physical system consisting of $N$ nonrelativistic electrons with spin- $\frac{1}{2}$ moving in an arbitrary external magnetic field $\vec{B}=\operatorname{curl} \vec{A}$, where $\vec{A}$ is the electromagnetic vector potential (with $\vec{\nabla} \cdot \vec{A}=0$ ), and in the electro-static field of $M$ nuclei with atomic numbers $Z_{j} \leq Z<\infty, j=1,2, \ldots, M$. Let $H_{Z, M, N}(\vec{A})$ denote the Hamiltonian of this system, which is a self-adjoint operator acting on the Hilbert space $\mathcal{H}_{N}$. The magnetic moment of the electron is proportional to its spin operator. The so-called gyro-magnetic factor, $g$, of the electron is the factor of proportionality between its spin and its magnetic moment; it has the value $g=2$, as correctly predicted by Dirac's equation for a relativistic electron. It had already been shown by Barry Simon et al. that if $g$ were smaller than 2 the system would be stable, in the sense that the corresponding Hamiltonian satisfies the lower bound

$$
H_{Z, M, N}(\vec{A})>-C Z^{2} N,
$$

uniformly in the vector potential $\vec{A}$, for a $g$-dependent finite constant $C>0$. Furthermore, if $g>2$ the system is unstable, even for a single electron, which is easy to verify. Nature wants us to understand whether the system is stable for $g=2$ !

E. H. Lieb, M. Loss, and H.-T. Yau, partly in collaboration with Jürg, proved in 1985 that for the physical value of the fine-structure constant $\alpha$, there is a critical value, $Z_{\text {crit }}$, of the atomic number of nuclei with the property that, for $Z>Z_{\text {crit }}$, the system is unstable even for only a single electron, quite a subtle result. However, at the time of Charlie's visit in Zurich, it was unknown whether, for the physical values of the $g$-factor and the fine-structure constant, i.e., for $g=2$ and $\alpha \simeq \frac{1}{137}$, stability of matter in magnetic fields holds, i.e., whether there exists a strictly positive $Z_{\text {crit }}$ such that, for $Z_{j} \leq Z<Z_{\text {crit }}, j=1, \ldots, M$, the system is stable, in the sense that

$$
H_{Z, M, N}(\vec{A})>-C_{Z}(N+M),
$$

for a finite constant $C_{Z}$, uniformly in $\vec{A}$, for an arbitrary number of electrons and nuclei. To make some relevant steps in the direction of establishing (18) was the project Jürg had proposed to his $\mathrm{PhD}$ student. The problem was well known to some of Jürg's friends at Princeton. When Charlie arrived in Zurich, Jürg had not advanced far in unraveling the mathematical subtleties hiding beneath (18)

\footnotetext{
${ }^{1}$ Maxi Seifert-nowadays an expert in the field of mathematical finance, the area that Luis also moved into after quantum mechanics.
} 
yet. But he knew that some of Charlie's techniques might be helpful and he explained the rather poor state of their understanding. Somewhat to his surprise, Charlie had never heard nor thought of this problem, but found the question worth thinking about. Two weeks after Charlie had returned to the US, he sent Jürg copies of hand-written notes containing a proof of (18) for $Z=1$, assuming that $\alpha$ is small enough. Jürg was deeply impressed by the ideas, which he found very beautiful, and even more by the enormous speed of Charlie's work. Needless to say, for Jürg and his student, this development was a mixed blessing.

Shortly after Charlie had found his proof of "Stability of matter in magnetic fields," Lieb, Loss, and Solovej discovered a considerably shorter and more elegant proof of this result, using one of Charlie's ideas. They proved the following variant of (18):

$$
H_{Z, M, N}>C_{Z} N^{1 / 3} M^{2 / 3},
$$

provided $\alpha<0.06$ and $Z \cdot \alpha^{2}<0.04$. Thus, the problem was settled!

Jürg also got more than his share of pleasure in studying systems of electrons and nuclei interacting with the electromagnetic field. For example, in a collaboration with Charlie, Gian Michele Graf, and a student, they showed that systems of electrons and nuclei interacting with the quantized electromagnetic field are stable, provided an ultraviolet cutoff is imposed on the electromagnetic field.

Charlie spent the academic year 2007-08 at Columbia University, during which he gave the Eilenberg Lectures on his seminal work on Whitney problems (described in Klartag's contribution). During this period, Charlie's and Michael's families befriended each other, and the conversations between Charlie and Michael ranged widely. Eventually they focused on mathematical aspects of an important problem in condensed matter physics: the properties of graphene and its artificial analogues.

Around that time Michael heard the physicists Horst Stoermer and Philip Kim lecture on the remarkable physical properties of graphene. Graphene is a nearly perfect two-dimensional material, a single atomic layer of carbon atoms arranged in a honeycomb structure. Just about every lecture on graphene by experimentalists and theorists begins with figures of its iconic Dirac cones, conical singularities in the band structure; see, for example, Figure 1. This structure is beautiful for its simplicity (generic band structures of crystals can be very messy) and profound in its physical implications.

To understand Dirac cones one considers the Schrödinger operator $H^{\lambda}=-\Delta+\lambda^{2} V(\mathbf{x})$, where $V$ is a honeycomb lattice potential. That is, $V$ is real-valued and periodic with respect to the equilateral triangular lattice $\Lambda$. Furthermore, with respect to some origin of coordinates in $\mathbb{R}^{2}, V$ is inversion symmetric (even) and $120^{\circ}$ rotationally invariant. An important example, corresponding to the single-electron model of graphene, is obtained by taking $V$ to be a sum of translates of a fixed atomic potential well, $V_{0}$, centered at the sites of a regular honeycomb structure. For any periodic potential,

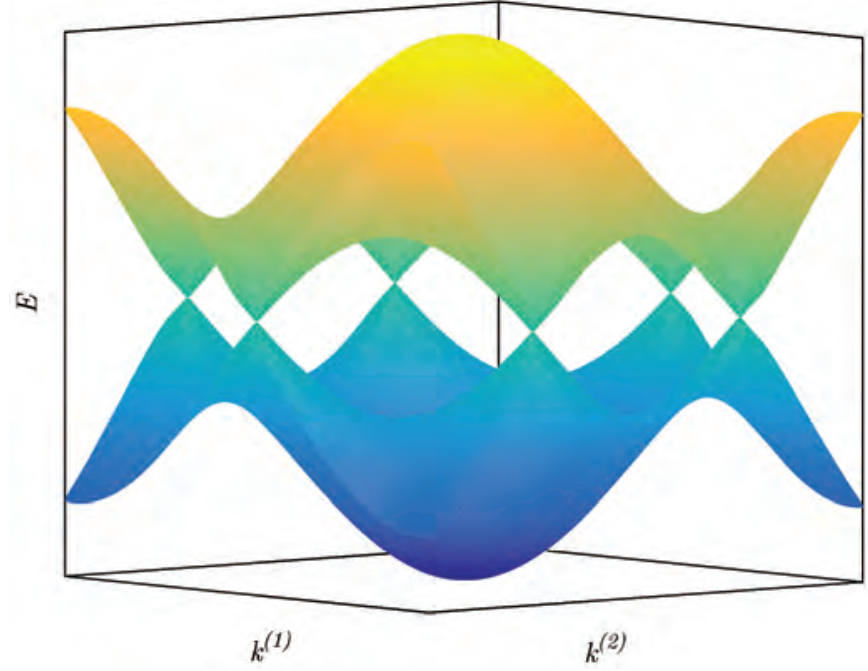

Figure 1. Dispersion surfaces of Wallace's celebrated 2-band tight-binding model with its conical singularities.

the $L^{2}$-spectrum is the union over quasi-momenta $\mathbf{k}$ in the Brillouin zone of the discrete (Floquet-Bloch) spectra of Hamiltonians $H^{\lambda}(\mathbf{k})=-(\nabla+i \mathbf{k})^{2}+\lambda^{2} V(\mathbf{x})$ on $L^{2}\left(\mathbb{R}^{2} / \Lambda\right)$ :

$$
E_{1}^{\lambda}(\mathbf{k}) \leq E_{2}^{\lambda}(\mathbf{k}) \leq \cdots \leq E_{b}^{\lambda}(\mathbf{k}) \leq \cdots .
$$

The maps $\mathbf{k} \mapsto E_{b}^{\lambda}(\mathbf{k})$ are Lipschitz continuous, and their graphs are called dispersion surfaces. Dirac points are energy-quasimomentum pairs $\left(E_{D}^{\lambda}, \mathbf{K}_{\star}\right)$ in a neighborhood of which two successive dispersion surfaces, $E_{-}^{\lambda}(\mathbf{k})$ and $E_{+}^{\lambda}(\mathbf{k})$, have the following behavior for $\mathbf{k}$ near $\mathbf{K}_{\star}$ :

$$
E_{ \pm}^{\lambda}(\mathbf{k})-E_{D}^{\lambda}= \pm v_{F}^{\lambda}\left|\mathbf{k}-\mathbf{K}_{\star}\right|\left(1+\mathcal{O}\left(\left|\mathbf{k}-\mathbf{K}_{\star}\right|\right)\right),
$$

where $v_{F}^{\lambda}>0$. The dispersion surfaces typically displayed by physicists are not of a Schrödinger operator on the continuum but rather the 2-band tight-binding (discrete) model of P. R. Wallace from his 1947 pioneering study of graphite; see Figure 1. This model is the workhorse for condensed matter modeling of graphene.

As their conversations meandered between math and non-math, Fefferman and Weinstein noted that all honeycomb Schrödinger operators with small (low contrast) potentials have Dirac points and Wallace's tight-binding model, likewise, has Dirac points. They wondered whether Dirac points exist for arbitrary, or at least generic, potentials of this class. In a joint 2012 paper in the Journal of the American Mathematical Society they proved that they do. Furthermore, for $V(\mathbf{x})$ equal to a sum of atomic potential wells centered on the honeycomb structure we have that $\left(E_{ \pm}^{\lambda}(\mathbf{k})-E_{D}^{\lambda}\right) / \nu_{F}^{\lambda}$ converges onto the two dispersion surfaces of Wallace's tight-binding model (Figure 1 ) as $\lambda$ tends to $\infty$ uniformly in $\mathbf{k}$ varying in the Brillouin zone, where $v_{F}^{\lambda} \approx e^{-c \lambda}>0$ depends on the atomic well.

They further explored the role that Dirac points play in the origin of robust (topologically protected) edge states, which are localized transverse to and plane-wave-like parallel to line defects through the honeycomb structure. 
Their remarkable stability and propagation properties are the hallmarks of topological insulators. Can one construct such states analytically? These questions led to a sequence of recent papers with J. P. Lee-Thorp; there remains a great deal to explore and understand in this very active area motivated by questions in condensed matter physics, photonics, and other fields.

These results are not the end of the story but are a promising beginning. During the past twenty-five years, many interesting results and novel analytical methods have been discovered. But some of the most challenging problems of quantum theory, e.g., the construction of a physically interesting four-dimensional, local, relativistic quantum field theory, in which Charlie's participation would undoubtedly have made a difference, remain open. We can always hope that Charlie will expand his engagement with the mathematics of quantum theory and fulfill Barry Simon's hope to become his secret weapon.

\begin{tabular}{|c|c|c|}
\hline \multicolumn{3}{|c|}{ Fefferman's PhD Students: } \\
\hline Antonio Córdoba & The University of Chicago & 1974 \\
\hline Elena Prestini & The University of Maryland & 1976 \\
\hline Bernard Marshall & Princeton University & 1977 \\
\hline Roberto Moriyon & Princeton University & 1979 \\
\hline Adrian Nachman & Princeton University & 1980 \\
\hline Antonio Sánchez Calle & e Princeton University & 1983 \\
\hline Barnwell Hughes & Princeton University & 1986 \\
\hline Matei Machedon & Princeton University & 1986 \\
\hline Joseph Gregg & Princeton University & 1987 \\
\hline Luis Seco & Princeton University & 1989 \\
\hline Alberto Parmeggiani & Princeton University & 1993 \\
\hline Jarosław Wróblewski & Princeton University & 1993 \\
\hline Alejandro Andreotti & Princeton University & 1994 \\
\hline Diego Córdoba & Princeton University & 1998 \\
\hline Ronald Howard & Princeton University & 1999 \\
\hline Alan Ho & Princeton University & 2001 \\
\hline Jorge Silva & Princeton University & 2001 \\
\hline Rami Shakarchi & Princeton University & 2002 \\
\hline Jose Rodrigo Diez & Princeton University & 2004 \\
\hline Spyros Alexakis & Princeton University & 2005 \\
\hline Garving Luli & Princeton University & 2010 \\
\hline Arie Israel & Princeton University & 2011 \\
\hline
\end{tabular}

\section{Image Credits}

Photo of C. Robin Graham, Charlie Fefferman, and Sun-

Yung Alice Chang courtesy of Sun-Yung Alice Chang.

All remaining photos and Figure 1 courtesy of Julie

Fefferman.

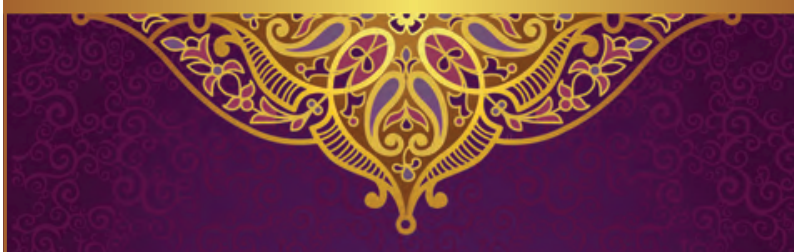

FEATURED TITLES FROM $\begin{array}{ll}\square \square \square & \text { HINDUSTAN } \\ \square \square & \text { BOOK AGENCY }\end{array}$

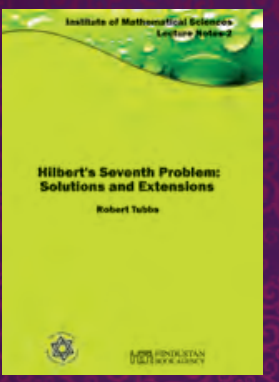

Hilbert's Seventh

Problem

Solutions and Extensions

Robert Tubbs, University of

Colorado, Boulder

This exposition is primarily a survey of the elementary yet subtle inno-

vations of several mathematicians between 1929 and 1934 that led to partial and then complete solutions to Hilbert's Seventh Problem (from the International Congress of Mathematicians in Paris, 1900).

2016; 94 pages; Softcover; ISBN: 978-93-80250-82-3; List US\$28; AMS members US $\$ 22.40$; Order code HIN/72

\section{Theory of Semigroups} and Applications

Kalyan B. Sinha, Jawaharlal Nehru Centre for Advanced Scientific

Research, Bangladore, India, and

Sachi Srivastava, University of Delhi South Campus, New Delhi, India

Combining the spirit of a textbook with that of a monograph on the topic of semigroups and their applications, this book will appeal to readers interested in operator the-

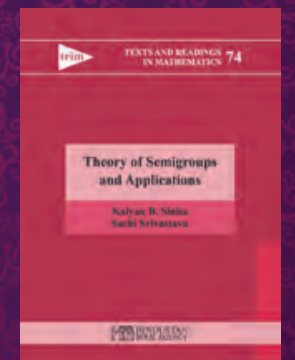
ory, partial differential equations, harmonic analysis, probability and statistics, and classical and quantum mechanics.

2017; 180 pages; Hardcover; ISBN: 978-93-86279-63-7; List US\$38; AMS members US $\$ 30.40$; Order code HIN/73

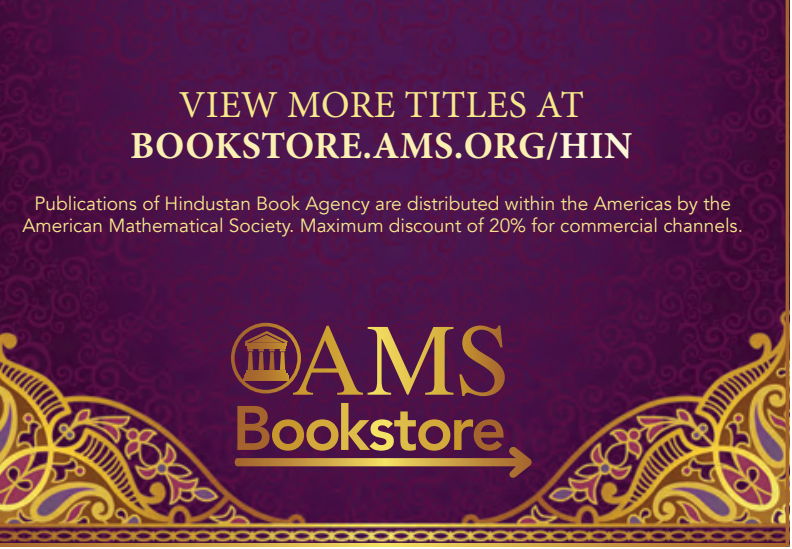

